\title{
SIGN PATTERNS ASSOCIATED WITH SOME GRAPHS THAT ALLOW OR REQUIRE DIAGONALIZABILITY*
}

\author{
SUNIL DAS ${ }^{\dagger}$
}

\begin{abstract}
The problems of characterizing sign pattern matrices that allow or require diagonalizability are mostly open. In this paper, we introduce the concept of essential index for a tree sign pattern matrix and use it to investigate the allow problem on diagonalizability for sign pattern matrices having their graphs as trees. We characterize sign pattern matrices allowing diagonalizability, whose graphs are star or path. We also give a sufficient condition for sign pattern matrices whose graphs are trees to allow diagonalizability. Further, we give a necessary condition for a sign pattern matrix to require diagonalizability and characterize all star sign pattern matrices that require diagonalizability.
\end{abstract}

Key words. Allow diagonalizability, Require diagonalizability, Tree, Sign pattern, Essential index.

AMS subject classifications. 15A20, 15B35, 05C50.

1. Introduction. A sign pattern matrix is a matrix with entries from $\{+,-, 0\}$. The qualitative class of a matrix $A$ is denoted by $Q(A)$ and is defined by the set of all real matrices with the same sign pattern as $A$. A sign pattern matrix $A$ allows a property $P$ if at least one matrix in $Q(A)$ has the property $P$ and requires a property $P$ if all matrices in $Q(A)$ have the property $P$. A square sign pattern matrix $A=\left[a_{i j}\right]$ is said to be combinatorially symmetric if for each $i, j$, either both $a_{i j}, a_{j i}$ are zero or both $a_{i j}, a_{j i}$ are nonzero.

Let us recall from [11] that the graph of a matrix $A$ of order $n$, denoted by $G(A)$, is defined to be a simple undirected graph with vertices $1,2, \ldots, n$ and for $i \neq j$ it has the edge $[i, j]$ if and only if $a_{i j} \neq 0$ or $a_{j i} \neq 0$. If this graph is a tree, then the matrix $A$ is irreducible if and only if $a_{i j} \neq 0$ whenever $a_{j i} \neq 0$. An irreducible sign pattern matrix whose graph is a tree is called a tree sign pattern matrix. Alternatively, a sign pattern matrix $A$ is a tree sign pattern matrix if $A$ is combinatorially symmetric and $G(A)$ is a tree. In particular, if $G(A)$ is a star or a path, then $A$ is called a star sign pattern matrix or a path sign pattern matrix, respectively.

Let $A=\left[a_{i j}\right]$ be a matrix of order $n$. The directed graph of $A$, denoted by $D(A)$, is defined as the directed graph with vertices $1,2, \ldots, n$ such that $D(A)$ has the arc $(i, j)$ from vertex $i$ to vertex $j$ if and only if $a_{i j} \neq 0$ (see [1, p. 29]). A nonzero product of the form $\gamma=a_{i_{1} i_{2}} a_{i_{2} i_{3}} \cdots a_{i_{k} i_{1}}$ in which the index set $\left\{i_{1}, i_{2}, \ldots, i_{k}\right\}$ consists of distinct indices is called a simple cycle of length $k$ (or simple $k$-cycle). A composite $k$-cycle is a product of simple cycles whose total length is $k$ and whose index sets are mutually disjoint. The maximum cycle length in $A$ is denoted by $c(A)$ (see [14]).

We recall from [10, Section 42.6] that the maximum rank of a sign pattern matrix $A$ is denoted by $\operatorname{MR}(A)$ and is defined by $\operatorname{MR}(A)=\max \{\operatorname{rank}(B): B \in Q(A)\}$.

Lemma $1.1([10])$. $\operatorname{MR}(A)$ is the maximum number of nonzero entries of $A$ no two of which are on the same row or the same column.

\footnotetext{
${ }^{*}$ Received by the editors on July 28, 2020. Accepted for publication on February 6, 2022. Handling Editor: Michael Tsatsomeros.

${ }^{\dagger}$ Department of Mathematics, Indian Institute of Technology Guwahati, Assam-781039, India (sunil.das@iitg.ac.in).
} 
Throughout this paper, $M_{n}$ denotes the set of all $n \times n$ real matrices. If $A \in M_{n}$, then $\sigma(A)$ denotes the set of all eigenvalues of $A, \sigma^{*}(A)$ denotes the set of all nonzero eigenvalues of $A, z(A)$ denotes the algebraic multiplicity of zero as an eigenvalue of $A$, and $P_{A}(x)$ denotes the characteristic polynomial of $A$ in $x$. If $\alpha$ and $\beta$ are subsets of $\{1,2, \ldots, n\}$, then $A[\alpha, \beta]$ is the submatrix of $A$ having rows and columns corresponding to the indices in $\alpha$ and $\beta$, respectively, and $A(\alpha, \beta)$ is the submatrix obtained from $A$ by deleting rows and columns corresponding to the indices in $\alpha$ and $\beta$, respectively. Further, $A[\alpha]=A[\alpha, \alpha]$ and $A(\alpha)=A(\alpha, \alpha)$.

In this paper, we attempt to address the problem of characterizing sign pattern matrices that allow or require diagonalizability when the associated graph is a special type of tree. In Section 2, we introduce the concept of essential index for a tree sign pattern matrix and find some results concerning the row spaces and column spaces of the matrices in its qualitative class. We use it in Section 3 to investigate the problem of allowing diagonalizability for sign pattern matrices $A$ (not necessarily combinatorially symmetric) when $G(A)$ is a tree and give a sufficient condition for $A$ to allow diagonalizability. When $G(A)$ is either a star or a path, we characterize sign pattern matrices $A$ allowing diagonalizability. Finally in Section 4 , we give a necessary condition for a sign pattern matrix to require diagonalizability and characterize all star sign pattern matrices that require diagonalizability.

2. Essential index. Let us recall that the standard determinant expansion of a square matrix $A=\left[a_{i j}\right]$ is

$$
\operatorname{det} A=\sum_{\sigma} \operatorname{sgn}(\sigma) a_{1 i_{1}} a_{2 i_{2}} \cdots a_{n i_{n}},
$$

where the summation extends over all permutations $\sigma=\left(i_{1}, i_{2}, \ldots, i_{n}\right)$ of $\{1,2, \ldots, n\}$ and $\operatorname{sgn}(\sigma)$ denotes the sign of the permutation $\sigma$. We have the following characterization of sign pattern matrices that require singularity.

Lemma $2.1([3,10])$. If $A$ is a sign pattern matrix of order $n$, then the following statements are equivalent.

1. A requires singularity.

2. Every term in the standard determinant expansion of $A$ is zero.

3. A has no composite n-cycle.

Feng et al. [8] showed that a sign pattern matrix $A$ allows diagonalizability if and only if there exists a matrix $B \in Q(A)$ such that $\operatorname{rank}(B)=\operatorname{rank}\left(B^{2}\right)$. Thus, if a sign pattern matrix $A$ does not allow diagonalizability, then $z(B) \geq 2$ for all $B \in Q(A)$. Further, Shao and Gao [14] proved that if $A$ is a combinatorially symmetric sign pattern matrix, then $A$ allows diagonalizability. Therefore, when $G(A)$ is a tree and $A$ is not combinatorially symmetric, the problem of $A$ to allow diagonalizability is worth to investigate if at least two irreducible components of $A$ require singularity. If $G(A)$ is a tree, then each irreducible component of $A$ is a tree sign pattern matrix. Moreover, for a tree sign pattern matrix $A$, the determinant of every $B \in Q(A)$ is a sum of products of entries of the forms $b_{i i}$ and $-b_{i j} b_{j i}$. So every term in the standard expansion of $B$ is 0 if some $b_{i i}$ s are 0 . The indices $i$ with $a_{i i}=0$ essential for $A$ to require singularity will be called essential indices. In Section 3, we will see that the problem of allowing diagonalizability has a relation with the connectivity of essential indices. Now we will give a formal definition of essential index.

A matching in an undirected graph is a set of edges in which no pair of edges shares a vertex. If $M$ is a matching in a graph $G$, then the vertices belonging to the edges of $M$ are said to be saturated by $M$ 
Electronic Journal of Linear Algebra, ISSN 1081-3810

A publication of the International Linear Algebra Society

Volume 38, pp. 131-159, February 2022.

(or $M$-saturated). Let $V(M)$ denotes the set of all $M$-saturated vertices in $G$. A maximum matching in a graph is a matching that has the largest possible cardinality. Throughout this section, $\langle n\rangle$ denotes the set $\{1,2, \ldots, n\}$.

Definition 2.2. Let $A$ be a tree sign pattern matrix of order $n$. An index $i$ is said to be an essential index of $A$ if there is a maximum matching $M$ in $G(A)$ such that $i \notin V(M)$ and every principal submatrix of $A$ whose index set contains $V(M) \cup\{i\}$ requires singularity.

An index $i$ is said to be nonessential if it is not an essential index. The following result clearly follows from Definition 2.2.

THEOREM 2.3. If $i$ is an essential index of a tree sign pattern matrix $A$, then $a_{i i}=0$.

The following example illustrates the essential and nonessential indices of a tree sign pattern matrix and establishes that the converse of Theorem 2.3 is not true.

ExAMPLE 2.4. Let us consider a tree sign pattern matrix $A$ with its graph $G(A)$ as follows.

$$
A=\left[\begin{array}{cccccc}
0 & 0 & - & 0 & 0 & 0 \\
0 & 0 & + & 0 & 0 & 0 \\
+ & - & + & + & 0 & 0 \\
0 & 0 & - & - & + & 0 \\
0 & 0 & 0 & + & + & + \\
0 & 0 & 0 & 0 & + & 0
\end{array}\right]
$$

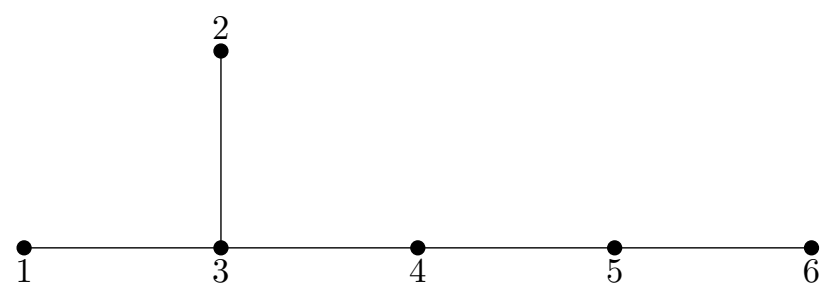

Since $a_{33}, a_{44}, a_{55} \neq 0$, by Theorem 2.3 , the indices 3,4 , and 5 are nonessential.

Let us consider a maximum matching $M_{1}=\{[2,3],[4,5]\}$ such that $1 \notin V\left(M_{1}\right)$. The subsets of $\langle 6\rangle$ which contain $V\left(M_{1}\right) \cup\{1\}$ are $\langle 5\rangle$ and $\langle 6\rangle$. Since both the principal submatrices $A[\langle 5\rangle]$ and $A[\langle 6\rangle]$ of $A$ require singularity, 1 is an essential index of $A$. Similarly, 2 is an essential index of $A$.

The maximum matchings that do not cover the vertex 6 are $M_{6}^{\prime}=\{[1,3],[4,5]\}$ and $M_{6}^{\prime \prime}=\{[2,3],[4,5]\}$. Let $S^{\prime}=V\left(M_{6}^{\prime}\right) \cup\{6\}=\{1,3,4,5,6\}$ and $S^{\prime \prime}=V\left(M_{6}^{\prime \prime}\right) \cup\{6\}=\{2,3,4,5,6\}$. Both the principal submatrices $A\left[S^{\prime}\right]$ and $A\left[S^{\prime \prime}\right]$ allow nonsingularity. So 6 is a nonessential index of $A$.

Therefore, the essential indices of $A$ are 1 and 2 , and the nonessential indices of $A$ are $3,4,5$, and 6 .

The main result of this section is given by the following theorem.

THEOREM 2.5. If a tree sign pattern matrix A of order $n$ requires singularity, then we have the following.

1. For each $B \in Q(A), \mathbf{e}_{k} \notin$ columnspace $(B)$ if $k$ is an essential index of $A$.

2. For each $B \in Q(A)$ with $\operatorname{rank}(B)=\operatorname{MR}(A), \mathbf{e}_{k} \in \operatorname{columnspace}(B)$ if $k$ is a nonessential index of $A$.

We prove Theorem 2.5 by using the following lemmas. Let us recall that if $G$ is a simple undirected graph, then $V(G)$ and $E(G)$ denote the vertex set of $G$ and the edge set of $G$, respectively. If $S \subseteq V(G)$, then $G-S$ is the subgraph of $G$ obtained from $G$ by deleting the vertices in $S$ and the edges incident to them. A path of length $k$ between two vertices $u$ and $v$ of $G$ is a sequence $\left\{v_{0}, v_{1}, \ldots, v_{k}\right\}$ of distinct vertices such that $v_{0}=u, v_{k}=v$, and $\left[v_{i-1}, v_{i}\right] \in E(G)$ for all $i \in\{1,2, \ldots, k\}$. A longest path is a path of maximum possible length. The degree of a vertex $u$, denoted by $\operatorname{deg}(u)$, is the number of distinct vertices of $G$ adjacent to $u$. If $\operatorname{deg}(u)=1$, then $u$ is called pendant. 
LEMMA 2.6. Let $A$ be a tree sign pattern matrix and suppose that $G(A)$ has vertices 1 and 2 , and let 2 be adjacent to 1 and $\operatorname{deg}(1)=1$. Then every maximum matching in $G(A)$ covers the vertex 2 , and thus 2 is a nonessential index of $A$.

Proof. Suppose that there exists a maximum matching $M$ in $G(A)$ such that $2 \notin V(M)$. Since $\operatorname{deg}(1)=1$ and 1 is adjacent to $2,1 \notin V(M)$. Then, $M \cup\{[1,2]\}$ is a matching in $G(A)$. This contradicts the assumption that $M$ is a maximum matching in $G(A)$. Therefore, every maximum matching in $G(A)$ covers the vertex 2 .

LEMma 2.7. Let $A$ be a tree sign pattern matrix. If $a_{i i}=0$ for at most one pendant vertex $i$ in $G(A)$, then $A$ allows nonsingularity.

Proof. Let $A$ be of order $n$ and $t$ be the number of vertices of degree $\geq 3$ in $G(A)$. We prove the result by induction on $t$. For $t=0, A$ is a path sign pattern matrix having a pendant vertex $i$ with $a_{i i} \neq 0$. So $A$ has a composite $n$-cycle and thus by Lemma 2.1, $A$ allows nonsingularity. So the result is true for $t=0$.

Suppose that $k \geq 1$ and the result is true for all $t<k$. Let $t=k$ and $A$ be a tree sign pattern matrix having $k$ vertices of degree $\geq 3$. Let $r$ be the pendant vertex such that $a_{r r}=0$, if it exists. Otherwise, consider any pendant vertex $r$. Let $\mathcal{P}$ be a longest path in $G(A)$ with an end vertex $r$. Then the other end vertex, say $s$, of $\mathcal{P}$ is also pendant in $G(A)$ so that $a_{s s} \neq 0$. Therefore, $A[V(\mathcal{P})]$ allows nonsingularity. Since $G(A)$ has at least one vertex of degree $\geq 3, \mathcal{P}$ covers at least one vertex of degree $\geq 3$. Therefore, each component of $G(A)-V(\mathcal{P})$ is a tree such that it has at most one pendant vertex $i$ with $a_{i i}=0$ and at most $k-1$ vertices of degree $\geq 3$. So by induction hypothesis, the principal submatrices corresponding to those components allow nonsingularity. Since $A(V(\mathcal{P}))$ is a direct sum of these principal submatrices, $A(V(\mathcal{P}))$ allows nonsingularity.

Since both $A[V(\mathcal{P})]$ and $A(V(\mathcal{P}))$ allow nonsingularity, by Lemma $2.1, A[V(\mathcal{P})]$ has a composite $|V(\mathcal{P})|-$ cycle and $A(V(\mathcal{P}))$ has a composite $(n-|V(\mathcal{P})|)$-cycle. Since the index sets of those two cycles are disjoint, the product of those two cycles is a composite $n$-cycle of $A$. Therefore, by Lemma $2.1, A$ does not require singularity, that is, $A$ allows nonsingularity.

Therefore, if a tree sign pattern matrix $A$ requires singularity, then $a_{i i}=0$ for at least two pendant vertices $i$ in $G(A)$.

LEMMA 2.8. Suppose that $A$ is a tree sign pattern matrix of order $n$ with $a_{n n}=0$, and $G(A)$ has vertices $n-1, n$ such that $n-1$ is adjacent to $n$ and $\operatorname{deg}(n)=1, \operatorname{deg}(n-1) \geq 2$. Let $i \in\{1,2, \ldots, n-2\}$. If $i$ is an essential index of $A$, then there exists a maximum matching $M$ in $G(A)$ such that $i \notin V(M), n \in V(M)$ and every principal submatrix of $A$ whose index set contains $V(M) \cup\{i\}$ requires singularity.

Proof. Since $i$ is an essential index of $A$, there exists a maximum matching $M$ in $G(A)$ such that $i \notin V(M)$ and every principal submatrix of $A$ whose index set contains $V(M) \cup\{i\}$ requires singularity. If $n \notin V(M)$, then by Lemma 2.6, $n-1 \in V(M)$. After a relabeling of the vertices $1,2, \ldots, n-2$, if required, we can assume that $[n-2, n-1] \in M$. Therefore, $i<n-2$. Since $M$ is a maximum matching in $G(A)$, $M^{\prime}=(M \backslash\{[n-2, n-1]\}) \cup\{[n-1, n]\}$ is a maximum matching in $G(A)$.

Let $T^{\prime}$ be such that $V\left(M^{\prime}\right) \cup\{i\} \subseteq T^{\prime} \subseteq\langle n\rangle$. If $n-2 \in T^{\prime}$, then $V(M) \cup\{i\} \subseteq T^{\prime}$. So $A\left[T^{\prime}\right]$ requires singularity. If $n-2 \notin T^{\prime}$, let $T=\left(T^{\prime} \backslash\{n\}\right) \cup\{n-2\}$. Then, $V(M) \cup\{i\} \subseteq T$ and thus $A[T]$ requires singularity. Now $T \backslash\{n-2, n-1\}=T^{\prime} \backslash\{n-1, n\} \subseteq\{1,2, \ldots, n-3\}, n-2 \notin T^{\prime}, n \notin T$ and $i<n-2$. If $Z=T \backslash\{n-2, n-1\}=T^{\prime} \backslash\{n-1, n\}$, then we have 


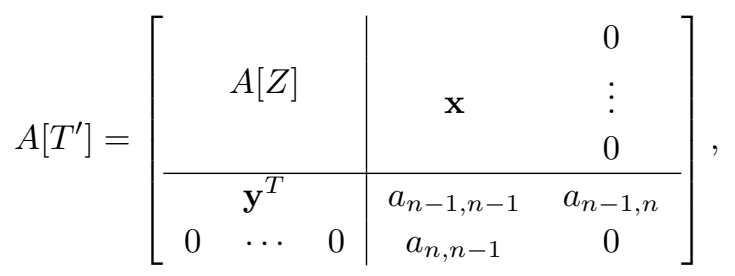

and

$$
A[T]=\left[\begin{array}{c|cc}
A[Z] & \mathbf{x}_{1} & \mathbf{x}_{2} \\
\hline \mathbf{y}_{1}^{T} & a_{n-2, n-2} & a_{n-2, n-1} \\
\mathbf{y}_{2}^{T} & a_{n-1, n-2} & a_{n-1, n-1}
\end{array}\right] .
$$

So $-a_{n-2, n-1} a_{n-1, n-2} \cdot \operatorname{det} A[Z]$ is a sum of some terms in the standard expansion of $\operatorname{det} A[T]$. Since $A[T]$ requires singularity, by Lemma 2.1, each term in the standard expansion of $\operatorname{det} A[T]$ is zero. Since $a_{n-2, n-1}, a_{n-1, n-2} \neq 0$, each term in the standard expansion of $\operatorname{det} A[Z]$ is zero.

Now each term in the standard expansion of $\operatorname{det} A\left[T^{\prime}\right]$ is a product of $-a_{n-1, n} a_{n, n-1}$ and a term in the standard expansion of $\operatorname{det} A[Z]$. Therefore, each term in the standard expansion of $\operatorname{det} A\left[T^{\prime}\right]$ is zero, and thus by Lemma 2.1, $A\left[T^{\prime}\right]$ requires singularity.

Hence, there exists a maximum matching $M^{\prime}$ in $G(A)$ such that $i \notin V\left(M^{\prime}\right), n \in V\left(M^{\prime}\right)$ and every principal submatrix of $A$ whose index set contains $V\left(M^{\prime}\right) \cup\{i\}$ requires singularity.

Lemma 2.9. Suppose that $A$ is a tree sign pattern matrix of order $n$, and $G(A)$ has vertices $n-2, n-1, n$ such that $n-1$ is adjacent to both $n-2, n$ and $\operatorname{deg}(n)=1, \operatorname{deg}(n-1)=2$. Let $a_{n n}=0, \tilde{A}=A[\langle n-2\rangle]$, and $G(\tilde{A})=G(A)-\{n-1, n\}$. Then, we have the following.

1. $n$ is an essential index of $A$ if and only if $n-2$ is an essential index of $A$.

2. For each $i \in\{1,2, \ldots, n-2\}, i$ is an essential index of $\tilde{A}$ if and only if $i$ is an essential index of $A$.

Proof. According to the given conditions, we can represent $G(A)$ as follows.

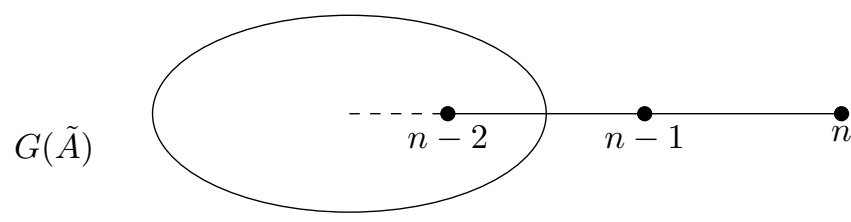

1. Let $n-2$ be an essential index of $A$. Then, there exists a maximum matching $M$ in $G(A)$ such that $n-2 \notin V(M)$ and every principal submatrix of $A$ whose index set contains $V(M) \cup\{n-2\}$ requires singularity. Since $M$ is a maximum matching in $G(A)$, therefore $[n-1, n] \in M$ and thus $\tilde{M}=(M \backslash\{[n-1, n]\}) \cup\{[n-2, n-1]\}$ is a maximum matching in $G(A)$. Since $V(\tilde{M}) \cup\{n\}=$ $V(M) \cup\{n-2\}, A[T]$ requires singularity for all $T$ satisfying $V(\tilde{M}) \cup\{n\} \subseteq T \subseteq\langle n\rangle$. Further, $n \notin V(\tilde{M})$. Therefore, $n$ is an essential index of $A$.

Similarly, if $n$ is an essential index of $A$, then $n-2$ is an essential index of $A$.

2. Here, $a_{n n}=0$. Let $T \subseteq\langle n\rangle$ be such that $n-1, n \in T$, and $\tilde{T}=T \backslash\{n-1, n\}$. Then, we can express $A[T]$ as: 


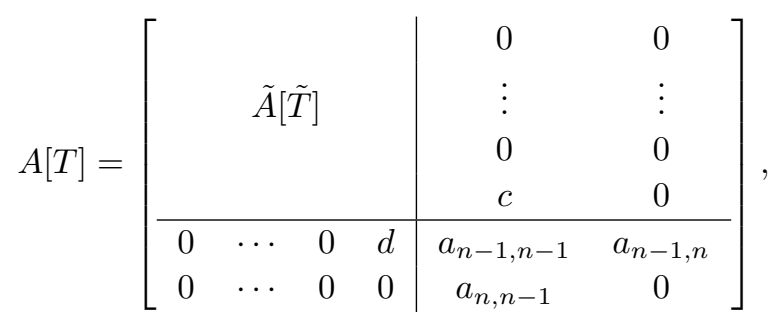

where $a_{n, n-1}, a_{n-1, n} \neq 0$, and either both $c, d$ are zero or both are nonzero. Thus, we have

$$
\operatorname{det} A[T]=-a_{n-1, n} a_{n, n-1} \cdot \operatorname{det} \tilde{A}[\tilde{T}]
$$

Suppose that $i$ is an essential index of $\tilde{A}$ for some $i<n-1$. Then, there exists a maximum matching $\tilde{M}$ in $G(\tilde{A})$ such that $i \notin V(\tilde{M})$ and $\tilde{A}[\tilde{T}]$ requires singularity for all $\tilde{T}$ satisfying $V(\tilde{M}) \cup\{i\} \subseteq \tilde{T} \subseteq\langle n-2\rangle$. Since $\tilde{M}$ is a maximum matching in $G(\tilde{A}), M=\tilde{M} \cup\{[n-1, n]\}$ is a maximum matching in $G(A)$. Let $T$ be such that $V(M) \cup\{i\} \subseteq T \subseteq\langle n\rangle$. Then by (2.3), $A[T]$ requires singularity. Further, $i \notin V(M)$. Therefore, $i$ is an essential index of $A$.

Conversely, suppose that $i$ is an essential index of $A$ for some $i<n-1$. Then there exists a maximum matching $M$ in $G(A)$ such that $i \notin V(M)$ and $A[T]$ requires singularity for all $T$ satisfying $V(M) \cup\{i\} \subseteq$ $T \subseteq\langle n\rangle$. By Lemma 2.8, we may assume without loss of generality that $n \in V(M)$. Then $[n-1, n] \in M$. Since $M$ is a maximum matching in $G(A), \tilde{M}=M \backslash\{[n-1, n]\}$ is a maximum matching in $G(\tilde{A})$. Let $\tilde{T}$ be such that $V(\tilde{M}) \cup\{i\} \subseteq \tilde{T} \subseteq\langle n-2\rangle$. Since $a_{n-1, n}, a_{n, n-1} \neq 0$, by (2.3), we can conclude that $\tilde{A}[\tilde{T}]$ requires singularity. Further, $i \notin V(\tilde{M})$. Therefore, $i$ is an essential index of $\tilde{A}$.

LEMMA 2.10. Suppose that $A$ is a tree sign pattern matrix of order $n$ with $a_{n n}=0$, and $G(A)$ has vertices $n-1, n$ such that $n-1$ is adjacent to $n$ and $\operatorname{deg}(n)=1, \operatorname{deg}(n-1) \geq 3$. Let the principal submatrices of $A$ corresponding to the components of $G(A)-\{n-1, n\}$ be $A_{1}, A_{2}, \ldots, A_{r}$.

1. If for some $t \in\{1,2, \ldots, r\}$, the vertices of $G\left(A_{t}\right)$ be $1,2, \ldots, k$ such that $k$ is adjacent to $n-1$, then we have the following.

(a) For each $i<k, i$ is an essential index of $A_{t}$ if and only if $i$ is an essential index of $A$.

(b) If $k$ is an essential index of $A_{t}$, then both $k$ and $n$ are essential indices of $A$.

(c) If $k$ is an essential index of $A$, then $k$ is an essential index of $A_{t}$.

2. If $n$ is an essential index of $A$, then there exists $l \in\{1,2, \ldots, r\}$ such that $p$ is an essential index of $A_{l}$, where $p$ is the vertex in $G\left(A_{l}\right)$ adjacent to $n-1$.

Proof. According to the given conditions, we can represent $G(A)$ as follows.

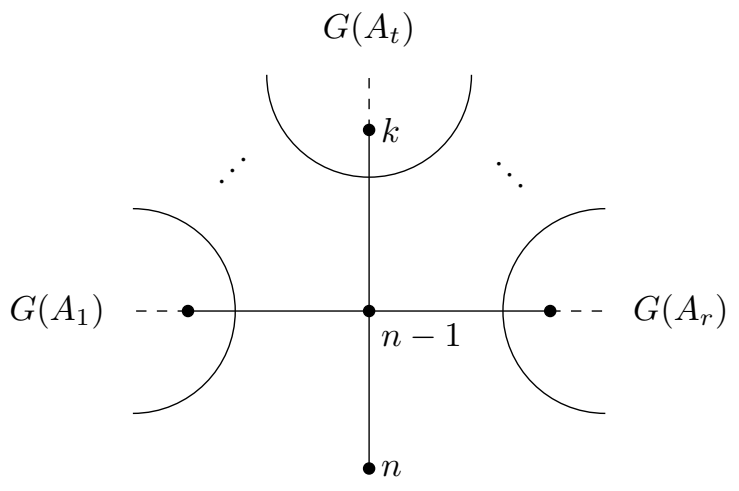


Electronic Journal of Linear Algebra, ISSN 1081-3810

A publication of the International Linear Algebra Society

Volume 38, pp. 131-159, February 2022.

Let the vertices of $G\left(A_{t}\right)$ be $1,2, \ldots, k$ such that $k$ is adjacent to $n-1$. Let $T \subseteq\langle n\rangle$ be such that $n-1, n \in T$. If $T_{t}=T \cap\langle k\rangle$ and $\tilde{T}=\{i \in T: k+1 \leq i \leq n-2\}$, then we can write $A[T]$ as:

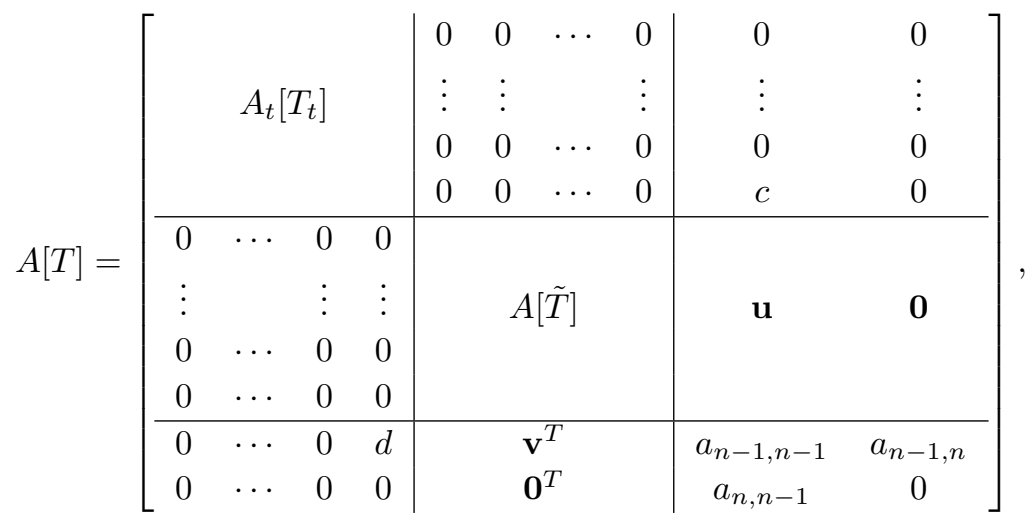

where either both $c$ and $d$ are zero or both are nonzero. Therefore,

$$
\operatorname{det} A[T]=-a_{n-1, n} a_{n, n-1} \cdot \operatorname{det} A_{t}\left[T_{t}\right] \cdot \operatorname{det} A[\tilde{T}] .
$$

If $\tilde{T}=V\left(M^{\prime}\right)$ for some matching $M^{\prime}$, then

$$
\operatorname{det} A[T]=-a_{n-1, n} a_{n, n-1} \cdot \operatorname{det} A_{t}\left[T_{t}\right]\left(\prod_{[i, j] \in M^{\prime}}\left(-a_{i j} a_{j i}\right)+\text { some more terms }\right) .
$$

1 (a). Suppose that $i$ is an essential index of $A_{t}$ for some $i<k$. Then there exists a maximum matching $M_{t}$ in $G\left(A_{t}\right)$ such that $i \notin V\left(M_{t}\right)$ and $A_{t}\left[T_{t}\right]$ requires singularity for all $T_{t}$ satisfying $V\left(M_{t}\right) \cup\{i\} \subseteq T_{t} \subseteq\langle k\rangle$. Let $M=\left(M_{1} \cup \cdots \cup M_{r}\right) \cup\{[n-1, n]\}$, where $M_{j}$ is a maximum matching in $G\left(A_{j}\right)$ for $j \neq t$ and $M_{t}$ is the maximum matching mentioned above. Then, $M$ is a maximum matching in $G(A)$ and $i \notin V(M)$. Let $T$ be such that $V(M) \cup\{i\} \subseteq T \subseteq\langle n\rangle$. If $T_{t}=T \cap\langle k\rangle$, then $A_{t}\left[T_{t}\right]$ requires singularity. Therefore, by (2.5), $A[T]$ requires singularity. Hence, $i$ is an essential index of $A$.

Conversely, suppose that $i$ is an essential index of $A$ for some $i<k$. Then, there exists a maximum matching $M$ in $G(A)$ such that $i \notin V(M)$ and $A[T]$ requires singularity for all $T$ satisfying $V(M) \cup\{i\} \subseteq$ $T \subseteq\langle n\rangle$. By Lemma 2.8, we can assume without loss of generality that $n \in V(M)$. Then, $[n-1, n] \in M$. Let $M_{j}=M \cap E\left(G\left(A_{j}\right)\right)$ for $j=1,2, \ldots, r$. Then, $M_{t}$ is a maximum matching in $G\left(A_{t}\right)$ and $i \notin V\left(M_{t}\right)$.

Let $T_{t}$ be such that $V\left(M_{t}\right) \cup\{i\} \subseteq T_{t} \subseteq\langle k\rangle$. If $T=T_{t} \cup\left(V\left(M_{1}\right) \cup \cdots \cup V\left(M_{r}\right)\right) \cup\{n-1, n\}$, then $A[T]$ requires singularity. Then each term in the standard expression of $A[T]$ is zero. Therefore, by $(2.6), A_{t}\left[T_{t}\right]$ requires singularity. Hence, $i$ is an essential index of $A_{t}$.

1(b). Suppose that $k$ is an essential index of $A_{t}$. Then there exists a maximum matching $M_{t}$ in $G\left(A_{t}\right)$ such that $k \notin V\left(M_{t}\right)$ and $A_{t}\left[T_{t}\right]$ requires singularity for all $T_{t}$ satisfying $V\left(M_{t}\right) \cup\{k\} \subseteq T_{t} \subseteq\langle k\rangle$. Let $M=\left(M_{1} \cup \cdots \cup M_{r}\right) \cup\{[n-1, n]\}$, where $M_{j}$ is a maximum matching in $G\left(A_{j}\right)$ for $j \neq t$ and $M_{t}$ is the above-mentioned matching. Then, $M$ is a maximum matching in $G(A)$ and $k \notin V(M)$. Therefore, $\tilde{M}=(M \backslash\{[n-1, n]\}) \cup\{[k, n-1]\}$ is also a maximum matching in $G(A)$ and $n \notin V(\tilde{M})$. Let $T$ be such that $V(M) \cup\{k\}=V(\tilde{M}) \cup\{n\} \subseteq T \subseteq\langle n\rangle$. If $T_{t}=T \cap\langle k\rangle$, then $A_{t}\left[T_{t}\right]$ requires singularity. Therefore by (2.5), $A[T]$ requires singularity. Hence, both $k$ and $n$ are essential indices of $A$. 
1(c). Suppose that $k$ is an essential index of $A$. Then by similar arguments as in the converse part of 1(a), $k$ is an essential index of $A_{t}$.

2. Suppose that there exist no $t \in\{1,2, \ldots, r\}$ such that $k_{t}$ is an essential index of $A_{t}$, where $k_{t}$ is a vertex of $G\left(A_{t}\right)$ adjacent to $n-1$. Let $M$ be a maximum matching in $G(A)$ such that $n \notin V(M)$. Since $n-1 \in V(M)$ (Lemma 2.6), there exists an $l \in\{1,2, \ldots, r\}$, where $G\left(A_{l}\right)$ (after a relabeling of vertices, if required) has vertices $1,2, \ldots, p$ with $p$ being adjacent to $n-1$ such that $[p, n-1] \in M$.

Since $M$ is a maximum matching in $G(A), \tilde{M}=(M \backslash\{[p, n-1]\}) \cup\{[n-1, n]\}$ is a maximum matching in $G(A)$. Therefore, $\tilde{M}_{l}=\tilde{M} \cap E\left(G\left(A_{l}\right)\right)$ is a maximum matching in $G\left(A_{l}\right)$. Since $p$ is a nonessential index of $A_{l}$ and $p \notin V\left(\tilde{M}_{l}\right)$, there exists $T_{l}$ satisfying $V\left(\tilde{M}_{l}\right) \cup\{p\} \subseteq T_{l} \subseteq\langle p\rangle$ such that $A_{l}\left[T_{l}\right]$ allows nonsingularity.

Let $M_{j}=M \cap E\left(G\left(A_{j}\right)\right)$ for $j=1,2, \ldots, r$. Then, $V\left(M_{l}\right)=V\left(\tilde{M}_{l}\right) \cup\{p\}$. If $T=T_{l} \cup\left(V\left(M_{1}\right) \cup \cdots \cup\right.$ $\left.V\left(M_{r}\right)\right) \cup\{n-1, n\}$, then $V(M) \cup\{n\} \subseteq T \subseteq\langle n\rangle$. Therefore by (2.6), $A[T]$ allows nonsingularity, which is a contradiction to the fact that $n$ is an essential index of $A$.

The following result confirms the existence of an essential index of a tree sign pattern matrix that requires singularity.

THEOREM 2.11. If a tree sign pattern matrix $A$ of order $n$ requires singularity, then $A$ has an essential index.

Proof. Suppose $A$ requires singularity. We prove the result by induction on $n$. For $n=1$, the result is obvious. If $n=2$, then $A$ allows nonsingularity. If $n=3$, then $A$ is given by:

$$
A=\left[\begin{array}{ccc}
0 & a_{12} & 0 \\
a_{21} & a_{22} & a_{23} \\
0 & a_{32} & 0
\end{array}\right],
$$

where $a_{12}, a_{21}, a_{23}, a_{32} \in\{+,-\}$. The maximum matching $M=\{[2,3]\}$ in $G(A)$ is such that $1 \notin V(M)$. The principal submatrix of $A$ whose index set contains $V(M) \cup\{1\}$ is $A$ itself, which requires singularity. Therefore, 1 is an essential index of $A$, and the result is true for $n=3$. Suppose the result is true for any $k<n$. Let $A$ be a tree sign pattern matrix of order $n$ requiring singularity. Then by Lemma 2.7 , we can assume without loss of generality that $a_{n n}=0$ and $\operatorname{deg}(n)=1$ in $G(A)$. Let $n$ be adjacent to $n-1$ in $G(A)$.

Case I: $\operatorname{deg}(n-1)=2$. Let $\tilde{A}=A[\langle n-2\rangle]$ and $G(\tilde{A})=G(A)-\{n-1, n\}$. Then, $\tilde{A}$ is a tree sign pattern matrix of order $n-2$ that requires singularity. By induction hypothesis, $\tilde{A}$ has an essential index, say $i$. Since $i \leq n-2$, by Lemma 2.9, we can conclude that $i$ is an essential index of $A$.

Case II: $\operatorname{deg}(n-1) \geq 3$. Let the principal submatrices of $A$ corresponding to the components of $G(A)-\{n-1, n\}$ be $A_{1}, A_{2}, \ldots, A_{r}$. Then there exists $t \in\{1,2, \ldots, r\}$ such that $A_{t}$ requires singularity. Let the vertices of $G\left(A_{t}\right)$ be $1,2, \ldots, k$ such that $k$ is adjacent to $n-1$ in $G(A)$. By induction hypothesis, $A_{t}$ has an essential index, say $i$. Therefore, by using Lemma 2.10, we can conclude that $i$ is an essential index of $A$.

Throughout the following discussion, $\mathbf{e}_{i}$ denotes the column vector whose $i$-th component is 1 and all other components are zero, $\mathbf{0}$ denotes the zero column vector, $O$ denotes the zero matrix, and their sizes will be clear from the context.

Lemma 2.12. Suppose that $A$ is a tree sign pattern matrix of order $n$, and $G(A)$ has vertices $n-2$, $n-1, n$ such that $n-1$ is adjacent to both $n-2, n$ and $\operatorname{deg}(n)=1, \operatorname{deg}(n-1)=2$. Let $a_{n n}=0$ and 
$\tilde{A}=A[\langle n-2\rangle]$. If $\mathbf{e}_{i} \in \operatorname{columnspace}(\tilde{B})$ for some $\tilde{B} \in Q(\tilde{A})$, then $\mathbf{e}_{i} \in \operatorname{columnspace}(B)$ for all $B \in Q(A)$ with $B[\langle n-2\rangle]=\tilde{B}$. Further, $\mathbf{e}_{n-2} \in \operatorname{columnspace}(\tilde{B})$ implies $\mathbf{e}_{n} \in \operatorname{columnspace}(B)$.

Proof. We can rewrite $A$ as:

$$
A=\left[\begin{array}{c|cc}
\tilde{A} & a_{n-2, n-1} \mathbf{e}_{n-2} & \mathbf{0} \\
\hline a_{n-1, n-2} \mathbf{e}_{n-2}^{T} & a_{n-1, n-1} & a_{n-1, n} \\
\mathbf{0}^{T} & a_{n, n-1} & 0
\end{array}\right],
$$

where $a_{n-1, n-2}, a_{n-2, n-1}, a_{n, n-1}, a_{n-1, n} \neq 0$.

Let $\mathbf{e}_{i} \in \operatorname{columnspace}(\tilde{B})$ for some $\tilde{B} \in Q(\tilde{A})$. Then there exists $\mathbf{p}_{i} \in \mathbb{R}^{n-2}$ such that $\tilde{B} \mathbf{p}_{i}=\mathbf{e}_{i}$.

Let $B \in Q(A)$ be such that $B[\langle n-2\rangle]=\tilde{B}$. Then there exist $b_{n-1, n}, b_{n, n-1} \neq 0$ such that

$$
B=\left[\begin{array}{c|cc}
\tilde{B} & b_{n-2, n-1} \mathbf{e}_{n-2} & \mathbf{0} \\
\hline b_{n-1, n-2} \mathbf{e}_{n-2}^{T} & b_{n-1, n-1} & b_{n-1, n} \\
\mathbf{0}^{T} & b_{n, n-1} & 0
\end{array}\right] .
$$

Therefore,

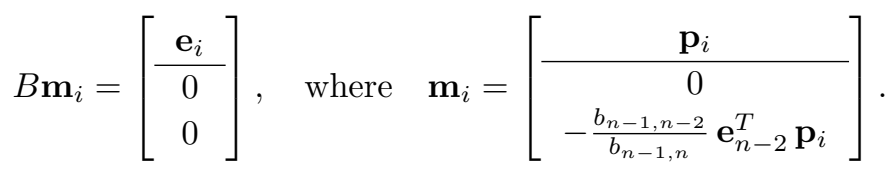

So $\mathbf{e}_{i} \in \operatorname{columnspace}(B)$.

Let $\mathbf{e}_{n-2} \in \operatorname{columnspace}(\tilde{B})$. Then there exists $\mathbf{p}_{n-2} \in \mathbb{R}^{n-2}$ such that $\tilde{B} \mathbf{p}_{n-2}=\mathbf{e}_{n-2}$. Therefore,

$$
B \mathbf{m}_{n-2}=\left[\begin{array}{c}
\mathbf{0} \\
0 \\
b_{n, n-1}
\end{array}\right] \text {, where } \mathbf{m}_{n-2}=\left[\frac{-b_{n-2, n-1} \mathbf{p}_{n-2}}{\frac{1}{\frac{1}{b_{n-1, n}}\left(b_{n-2, n-1} b_{n-1, n-2} \mathbf{e}_{n-2}^{T} \mathbf{p}_{n-2}-b_{n-1, n-1}\right)}}\right] .
$$

So $\mathbf{e}_{n} \in \operatorname{columnspace}(B)$.

Lemma 2.13. Suppose that $A$ is a tree sign pattern matrix of order $n$ with $a_{n n}=0$, and $G(A)$ has vertices $n-1, n$ such that $n-1$ is adjacent to $n$ and $\operatorname{deg}(n)=1, \operatorname{deg}(n-1) \geq 3$. Let the principal submatrix corresponding to one component of $G(A)-\{n-1, n\}$ be $\tilde{A}$ such that $G(\tilde{A})$ has the vertices $1,2, \ldots, k$ with $k$ being adjacent to $n-1$. If $\mathbf{e}_{i} \in \operatorname{columnspace}(\tilde{B})$ for some $\tilde{B} \in Q(\tilde{A})$, then for each $B \in Q(A)$ with $B[\langle k\rangle]=\tilde{B}, \mathbf{e}_{i} \in \operatorname{columnspace}(B)$.

Proof. According to the given conditions, we can express $A$ as:

$$
A=\left[\begin{array}{cccc}
\tilde{A} & O & a_{k, n-1} \mathbf{e}_{k} & \mathbf{0} \\
O & A[\alpha] & \mathbf{u} & \mathbf{0} \\
a_{n-1, k} \mathbf{e}_{k}^{T} & \mathbf{v}^{T} & a_{n-1, n-1} & a_{n-1, n} \\
\mathbf{0}^{T} & \mathbf{0}^{T} & a_{n, n-1} & 0
\end{array}\right],
$$

where $\alpha=\{k+1, k+2, \ldots, n-2\}, \mathbf{u}, \mathbf{v} \neq \mathbf{0}$ and $a_{n-1, k}, a_{k, n-1}, a_{n-1, n}, a_{n, n-1} \neq 0$.

Let $\tilde{B} \in Q(\tilde{A})$ be such that $\mathbf{e}_{i} \in \operatorname{columnspace}(\tilde{B})$. Then there exists a vector $\mathbf{p}_{i} \in \mathbb{R}^{k}$ such that $\tilde{B} \mathbf{p}_{i}=\mathbf{e}_{i}$.

Let $B \in Q(A)$ be such that $B[\langle k\rangle]=\tilde{B}$. Then there exist $b_{n-1, n} \neq 0$ such that

$$
B=\left[\begin{array}{cccc}
\tilde{B} & O & b_{k, n-1} \mathbf{e}_{k} & \mathbf{0} \\
O & \widehat{B} & \mathbf{x} & \mathbf{0} \\
b_{n-1, k} \mathbf{e}_{k}^{T} & \mathbf{y}^{T} & b_{n-1, n-1} & b_{n-1, n} \\
\mathbf{0}^{T} & \mathbf{0}^{T} & b_{n, n-1} & 0
\end{array}\right] .
$$


Therefore,

$$
B \mathbf{m}_{i}=\left[\begin{array}{c}
\mathbf{e}_{i} \\
\mathbf{0} \\
0 \\
0
\end{array}\right], \quad \text { where } \quad \mathbf{m}_{i}=\left[\begin{array}{c}
\mathbf{p}_{i} \\
\mathbf{0} \\
0 \\
-\frac{b_{n-1, k}}{b_{n-1, n}} \mathbf{e}_{k}^{T} \mathbf{p}_{i}
\end{array}\right] .
$$

Therefore, for every $B \in Q(A)$ with $B[\langle k\rangle]=\tilde{B}, \mathbf{e}_{i} \in \operatorname{columnspace}(B)$.

Next, we prove both parts of Theorem 2.5 separately.

Proof of Theorem 2.5.1. We prove the result by induction on $n$.

Induction base case. For $n=1$, the result is obvious. If $n=2$, then $A$ allows nonsingularity. For $n=3$, since $A$ requires singularity, $A$ is given by:

$$
A=\left[\begin{array}{ccc}
0 & a_{12} & 0 \\
a_{21} & a_{22} & a_{23} \\
0 & a_{32} & 0
\end{array}\right]
$$

where $a_{12}, a_{21}, a_{23}, a_{32} \in\{+,-\}$. The set of all essential indices of $A$ is $\{1,3\}$. Clearly, for every $B \in Q(A)$, $\mathbf{e}_{1}, \mathbf{e}_{3} \notin$ columnspace $(B)$. Thus, the result is also true for $n=3$.

Induction hypothesis. Suppose that the result is true for any tree sign pattern matrix of order less than $n$.

Induction final step. Let $A$ be a tree sign pattern matrix of order $n$ such that $A$ requires singularity. Then by Lemma 2.7, there exist vertices in $G(A)$, say $n-1, n$, such that $n$ is adjacent to $n-1, a_{n n}=0$, and $\operatorname{deg}(n)=1$.

Case I: $\operatorname{deg}(n-1)=2$. Let $n-2$ be adjacent to $n-1$. Then, we can rewrite $A$ as:

$$
A=\left[\begin{array}{ccc}
\tilde{A} & a_{n-2, n-1} \mathbf{e}_{n-2} & \mathbf{0} \\
a_{n-1, n-2} \mathbf{e}_{n-2}^{T} & a_{n-1, n-1} & a_{n-1, n} \\
\mathbf{0}^{T} & a_{n, n-1} & 0
\end{array}\right]
$$

where $\tilde{A}=A[\langle n-2\rangle]$ and $a_{n-1, n}, a_{n, n-1}, a_{n-1, n-2}, a_{n-2, n-1} \neq 0$.

Let $n$ be an essential index of $A$. Then by Lemma 2.9, n-2 is an essential index of $\tilde{A}$ and hence by induction hypothesis, for every $\tilde{B} \in Q(\tilde{A}), \mathbf{e}_{n-2} \notin \operatorname{columnspace}(\tilde{B})$. If $\mathbf{e}_{n} \in \operatorname{columnspace}(B)$ for some $B \in Q(A)$, then there exist $c_{n-1}, c_{n} \in \mathbb{R}$ and $\mathbf{p} \in \mathbb{R}^{n-2}$ such that

$$
\left[\begin{array}{l}
\mathbf{0} \\
0 \\
1
\end{array}\right]=\left[\begin{array}{c}
\tilde{B} \\
b_{n-1, n-2} \mathbf{e}_{n-2}^{T} \\
\mathbf{0}^{T}
\end{array}\right] \mathbf{p}+c_{n-1}\left[\begin{array}{c}
b_{n-2, n-1} \mathbf{e}_{n-2} \\
b_{n-1, n-1} \\
b_{n, n-1}
\end{array}\right]+c_{n}\left[\begin{array}{c}
\mathbf{0} \\
b_{n-1, n} \\
0
\end{array}\right],
$$

where $\tilde{B}=B[\langle n-2\rangle] \in Q(\tilde{A})$.

Since $c_{n-1}, b_{n-2, n-1} \neq 0$ and $\tilde{B} \mathbf{p}=-c_{n-1} b_{n-2, n-1} \mathbf{e}_{n-2}, \mathbf{e}_{n-2} \in \operatorname{columnspace}(\tilde{B})$, which is a contradiction. 
Electronic Journal of Linear Algebra, ISSN 1081-3810

A publication of the International Linear Algebra Society

Volume 38, pp. 131-159, February 2022.

Let $i(\neq n)$ be an essential index of $A$. Then by Lemma 2.6, $i \neq n-1$, and by Lemma 2.9, $i$ is an essential index of $\tilde{A}$. If $\mathbf{e}_{i} \in \operatorname{columnspace}(B)$ for some $B \in Q(A)$, then from (2.7) we can conclude that $\mathbf{e}_{i}$ is a linear combination of all but the $(n-1)$-th column of $B$. Then for some $a_{i} \in \mathbb{R}, \mathbf{e}_{i}+a_{i} \mathbf{e}_{n-1}$ is a linear combination of the first $n-2$ columns of $B$ and thus $\mathbf{e}_{i} \in \operatorname{columnspace}(\tilde{B})$, where $\tilde{B}=B[\langle n-2\rangle] \in Q(\tilde{A})$. This is a contradiction to the induction hypothesis.

Case II: $\operatorname{deg}(n-1) \geq 3$. Suppose that $G(A)-\{n-1, n\}$ has $r$ components. Then, $A[\langle n-2\rangle]$ is a direct sum of $r$ irreducible components, say $A_{1}, A_{2}, \ldots, A_{r}$.

Let $n$ be an essential index of $A$. Then by Lemma 2.10, there exists $t \in\{1,2, \ldots, r\}$ such that $k$ is an essential index of $A_{t}$, where $k$ is the vertex of $G\left(A_{t}\right)$ adjacent to $n-1$. By induction hypothesis, for every $B_{t} \in Q\left(A_{t}\right), \mathbf{e}_{k} \notin \operatorname{columnspace}\left(B_{t}\right)$. Without loss of generality, let us assume that $G\left(A_{t}\right)$ has vertices $1,2, \ldots, k$. So we can write $A$ as:

$$
A=\left[\begin{array}{cccc}
A_{t} & \mathbf{0} & a_{k, n-1} \mathbf{e}_{k} & \mathbf{0} \\
\mathbf{0}^{T} & A[\alpha] & \mathbf{u} & \mathbf{0} \\
a_{n-1, k} \mathbf{e}_{k}^{T} & \mathbf{v}^{T} & a_{n-1, n-1} & a_{n-1, n} \\
\mathbf{0}^{T} & \mathbf{0}^{T} & a_{n, n-1} & 0
\end{array}\right]
$$

where $\alpha=\{k+1, \ldots, n-2\}, a_{n-1, k}, a_{k, n-1}, a_{n-1, n}, a_{n, n-1} \neq 0$ and $\mathbf{u}, \mathbf{v} \neq \mathbf{0}$. If $\mathbf{e}_{n} \in \operatorname{columnspace}(B)$ for some $B \in Q(A)$, then by similar arguments as provided in Case I, $\mathbf{e}_{k} \in \operatorname{columnspace}\left(B_{t}\right)$, where $B_{t}=B[\langle k\rangle] \in Q\left(A_{t}\right)$. This is a contradiction to the induction hypothesis.

Let $i(\neq n)$ be an essential index of $A$. Then by Lemma 2.6, $i \neq n-1$. Without loss of generality, we may assume that $i$ is a vertex of $G\left(A_{t}\right)$ and the vertices of $G\left(A_{t}\right)$ are $1,2, \ldots, k$. Then by Lemma $2.10, i$ is an essential index of $A_{t}$. If $\mathbf{e}_{i} \in \operatorname{columnspace}(B)$ for some $B \in Q(A)$, then from (2.8) we can conclude that $\mathbf{e}_{i}$ is a linear combination of the first $k$ columns and the $n$-th column of $B$. Then for some $a_{i} \in \mathbb{R}, \mathbf{e}_{i}+a_{i} \mathbf{e}_{n-1}$ is a linear combination of the first $k$ columns of $B$ and thus $\mathbf{e}_{i} \in \operatorname{columnspace}\left(B_{t}\right)$, where $B_{t}=B[\langle k\rangle] \in Q\left(A_{t}\right)$. This is a contradiction to the induction hypothesis.

Proof of Theorem 2.5.2. We prove the result by induction on $n$.

Induction base case. For $n=1$, the result is obvious. If $n=2$, then $A$ allows nonsingularity. For $n=3$, since $A$ requires singularity, $A$ is given by:

$$
A=\left[\begin{array}{ccc}
0 & a_{12} & 0 \\
a_{21} & a_{22} & a_{23} \\
0 & a_{32} & 0
\end{array}\right],
$$

where $a_{12}, a_{21}, a_{23}, a_{32} \in\{+,-\}$. The only nonessential index of $A$ is 2 . Clearly, $\mathbf{e}_{2} \in \operatorname{columnspace}(B)$ and $\operatorname{rank}(B)=2=\operatorname{MR}(A)$ for all $B \in Q(A)$. Thus, the result is also true for $n=3$.

Induction hypothesis. Suppose that the result is true for any tree sign pattern matrix of order less than $n$.

Induction final step. Let $A$ be a tree sign pattern matrix of order $n$ such that $A$ requires singularity. Then by Lemma 2.7, there exist vertices in $G(A)$, say $n-1, n$, such that $n$ is adjacent to $n-1, a_{n n}=0$ and $\operatorname{deg}(n)=1$. 
Case I: $\operatorname{deg}(n-1)=2$. Let $n-2$ be adjacent to $n-1$. Then, we can rewrite $A$ as:

$$
A=\left[\begin{array}{ccc}
\tilde{A} & a_{n-2, n-1} \mathbf{e}_{n-2} & \mathbf{0} \\
a_{n-1, n-2} \mathbf{e}_{n-2}^{T} & a_{n-1, n-1} & a_{n-1, n} \\
\mathbf{0}^{T} & a_{n, n-1} & 0
\end{array}\right],
$$

where $\tilde{A}=A[\langle n-2\rangle]$ and $a_{n-1, n}, a_{n, n-1}, a_{n-1, n-2}, a_{n-2, n-1} \neq 0$. By Lemma 1.1, $\operatorname{MR}(A)=\operatorname{MR}(\tilde{A})+2$.

Let $N$ be the set of all nonessential indices of $\tilde{A}$. Then from Lemma 2.9, we can conclude that the set of all nonessential indices of $A$ is given by either $N \cup\{n-1, n\}$ or $N \cup\{n-1\}$ accordingly as $n-2 \in N$ or $n-2 \notin N$.

Let $B \in Q(A)$ be such that $\operatorname{rank}(B)=\mathrm{MR}(A)$. Then

$$
B=\left[\begin{array}{c|cc}
\tilde{B} & b_{n-2, n-1} \mathbf{e}_{n-2} & \mathbf{0} \\
\hline b_{n-1, n-2} \mathbf{e}_{n-2}^{T} & b_{n-1, n-1} & b_{n-1, n} \\
\mathbf{0}^{T} & b_{n, n-1} & 0
\end{array}\right],
$$

where $b_{n-1, n}, \quad b_{n, n-1} \neq 0$, and $\tilde{B}=B[\langle n-2\rangle] \in Q(\tilde{A})$. It can be easily verified that $\operatorname{rank}(\tilde{B})=\operatorname{rank}(B)-$ $2=\operatorname{MR}(A)-2=\operatorname{MR}(\tilde{A})$. Therefore, by induction hypothesis, $\mathbf{e}_{i} \in \operatorname{columnspace}(\tilde{B})$ for all $i \in N$. Hence, by Lemma 2.12, $\mathbf{e}_{i} \in \operatorname{columnspace}(B)$ for all $i \in N$. Since $B \mathbf{e}_{n}=b_{n-1, n} \mathbf{e}_{n-1}$, therefore $\mathbf{e}_{n-1} \in$ columnspace $(B)$.

Suppose that $n-2 \in N$. Then, $\mathbf{e}_{n-2} \in \operatorname{columnspace}(\tilde{B})$. Therefore, by Lemma $2.12, \mathbf{e}_{n} \in$ $\operatorname{columnspace}(B)$.

Therefore, for each $B \in Q(A)$ with $\operatorname{rank}(B)=\operatorname{MR}(A), \mathbf{e}_{i} \in \operatorname{columnspace}(B)$ for all nonessential indices $i$ of $A$.

Case II: $\operatorname{deg}(n-1) \geq 3$. Suppose that $G(A)-\{n-1, n\}$ has $r$ components. Then, $A[\langle n-2\rangle]$ is a direct sum of $r$ irreducible components, say $A_{1}, A_{2}, \ldots, A_{r}$.

Let the vertices of $G\left(A_{1}\right), \ldots, G\left(A_{r}\right)$ adjacent to $n-1$ be $k_{1}, \ldots, k_{r}$, respectively. Then, $A$ can be expressed as:

$$
A=\left[\begin{array}{cccc|cc}
A_{1} & O & \cdots & O & & \\
O & A_{2} & \ddots & \vdots & \mathbf{u} & \mathbf{0} \\
\vdots & \ddots & \ddots & O & & \\
O & \cdots & O & A_{r} & & \\
\hline & \mathbf{v}^{T} & & a_{n-1, n-1} & a_{n-1, n} \\
& \mathbf{0}^{T} & & a_{n, n-1} & 0
\end{array}\right]
$$

where $\mathbf{u}=a_{k_{1}, n-1} \mathbf{e}_{k_{1}}+\cdots+a_{k_{r}, n-1} \mathbf{e}_{k_{r}}, \mathbf{v}=a_{n-1, k_{1}} \mathbf{e}_{k_{1}}+\cdots+a_{n-1, k_{r}} \mathbf{e}_{k_{r}}$ for some $a_{k_{1}, n-1}, a_{n-1, k_{1}}, \ldots$ $a_{k_{r}, n-1}, a_{n-1, k_{r}} \neq 0$, and $a_{n-1, n}, a_{n, n-1} \neq 0$. Therefore, by Lemma 1.1, $\operatorname{MR}(A)=\operatorname{MR}\left(A_{1}\right)+\cdots+\operatorname{MR}\left(A_{r}\right)+2$. 
Electronic Journal of Linear Algebra, ISSN 1081-3810

A publication of the International Linear Algebra Society

Volume 38, pp. 131-159, February 2022.

Let $B \in Q(A)$ be such that $\operatorname{rank}(B)=\mathrm{MR}(A)$. Then there exist $b_{n-1, n}, b_{n, n-1} \neq 0$ such that

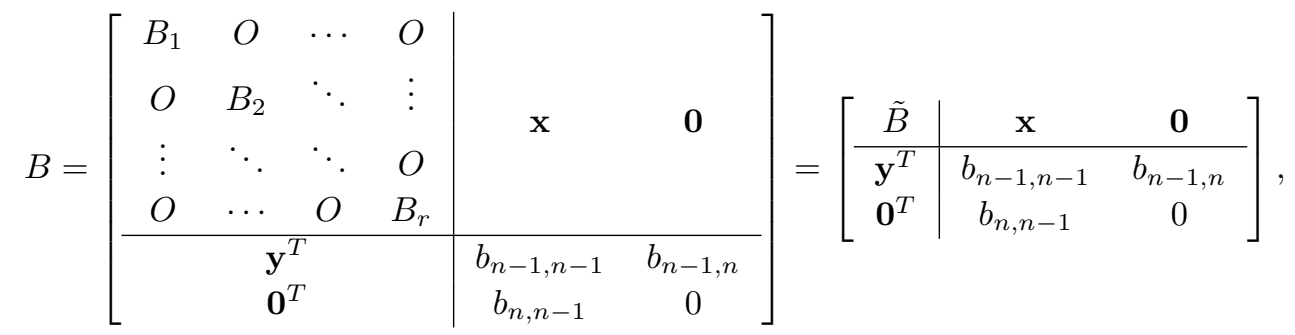

where $\tilde{B}=B[\langle n-2\rangle]$ and $B_{t} \in Q\left(A_{t}\right)$ for $t=1,2, \ldots, r$. It can be easily verified that

$$
\operatorname{rank}(\tilde{B})=\operatorname{rank}(B)-2=\operatorname{MR}(A)-2=\operatorname{MR}\left(A_{1}\right)+\cdots+\operatorname{MR}\left(A_{r}\right),
$$

and thus $\operatorname{rank}\left(B_{t}\right)=\operatorname{MR}\left(A_{t}\right)$ for $t=1,2, \ldots, r$.

If $N=\left\{i: i\right.$ is a nonessential index of $A_{t}$ for some $\left.t \in\{1,2, \ldots, r\}\right\}$, then the set of all nonessential indices of $A$ is either $N \cup\{n-1\}$ or $N \cup\{n-1, n\}$.

If $A_{t}$ allows nonsingularity, then $B_{t}$ is invertible, and thus $\mathbf{e}_{i} \in \operatorname{columnspace}\left(B_{t}\right)$ for all indices $i$ of $A_{t}$. Further, by Definition 2.2, each index of $A_{t}$ is nonessential. If $A_{t}$ requires singularity, then by induction hypothesis $\mathbf{e}_{i} \in \operatorname{columnspace}\left(B_{t}\right)$ for all nonessential indices $i$ of $A_{t}$. Now by Lemma 2.10 , all nonessential indices of $A_{t}$ are also nonessential for $A$. Therefore, using Lemma 2.13, we can conclude that $\mathbf{e}_{i} \in \operatorname{columnspace}(B)$ for all nonessential indices $i(<n-1)$ of $A$. Further, $B \mathbf{e}_{n}=b_{n-1, n} \mathbf{e}_{n-1}$ implies $\mathbf{e}_{n-1} \in \operatorname{columnspace}(B)$.

If $n$ is a nonessential index of $A$, then by Lemma $2.10 .1(\mathrm{~b}), k_{1}, \ldots, k_{r}$ are nonessential indices of $A_{1}, \ldots, A_{r}$, respectively. So by Lemma $2.13, \mathbf{e}_{i} \in \operatorname{columnspace}(\tilde{B})$ for $i=k_{1}, k_{2}, \ldots, k_{r}$. Since $\mathbf{x} \in Q(\mathbf{u})$, there exist nonzero $b_{k_{1}, n-1}, \ldots, b_{k_{r}, n-1}$ such that $\mathbf{x}=b_{k_{1}, n-1} \mathbf{e}_{k_{1}}+\cdots+b_{k_{r}, n-1} \mathbf{e}_{k_{r}}$. So $\mathbf{x} \in \operatorname{columnspace}(\tilde{B})$. Therefore, there exists $\mathbf{p} \in \mathbb{R}^{n-2}$ such that $\tilde{B} \mathbf{p}=\mathbf{x}$. Therefore,

$$
B \mathbf{m}=\left[\begin{array}{c}
\mathbf{0} \\
0 \\
b_{n, n-1}
\end{array}\right], \quad \text { where } \mathbf{m}=\left[\frac{-\mathbf{p}}{1} \frac{\mathbf{y}^{T} \mathbf{p}-b_{n-1, n-1}}{b_{n-1, n}}\right] .
$$

So $\mathbf{e}_{n} \in \operatorname{columnspace}(B)$. Hence, for each $B \in Q(A)$ with $\operatorname{rank}(B)=\operatorname{MR}(A), \mathbf{e}_{i} \in \operatorname{columnspace}(B)$ for all nonessential indices $i$ of $A$.

The following example illustrates the necessity of maximum rank in the above theorem.

EXAMPLE 2.14. Let us consider a tree sign pattern matrix $A$ with its graph $G(A)$ as follows.

$$
A=\left[\begin{array}{ccccc}
0 & + & + & + & 0 \\
- & 0 & 0 & 0 & 0 \\
+ & 0 & 0 & 0 & 0 \\
+ & 0 & 0 & - & - \\
0 & 0 & 0 & - & -
\end{array}\right]
$$

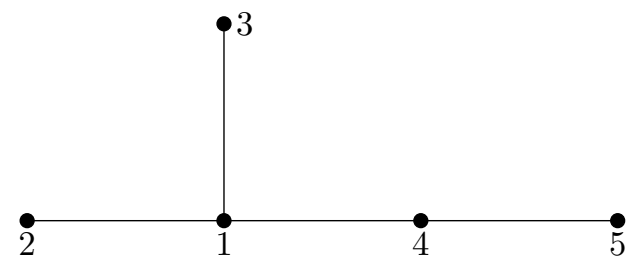

Now $M_{2}=\{[1,3],[4,5]\}$ and $M_{3}=\{[1,2],[4,5]\}$ are maximum matchings such that $2 \notin V\left(M_{2}\right)$ and $3 \notin V\left(M_{3}\right)$. Since $V\left(M_{2}\right) \cup\{2\}=V\left(M_{3}\right) \cup\{3\}=\langle 5\rangle$ and $A$ requires singularity, both 2,3 are essential 
indices of $A$. The nonessential indices of $A$ are $1,4,5$, since every maximum matching saturates the vertices $1,4,5$. Let

$$
B=\left[\begin{array}{ccccc}
0 & 2 & 1 & 3 & 0 \\
-3 & 0 & 0 & 0 & 0 \\
1 & 0 & 0 & 0 & 0 \\
5 & 0 & 0 & -2 & -2 \\
0 & 0 & 0 & -2 & -2
\end{array}\right] \in Q(A)
$$

Then $\operatorname{rank}(B)=3<4=\operatorname{MR}(A)$. Further, $\mathbf{e}_{4}, \mathbf{e}_{5} \notin$ columnspace $(B)$.

Since $A$ is combinatorially symmetric, we have the following corollary.

COROLlary 2.15. If a tree sign pattern matrix $A$ of order $n$ requires singularity, then we have the following.

1. For each $B \in Q(A), \mathbf{e}_{k} \notin \operatorname{rowspace}(B)$ if $k$ is an essential index of $A$.

2. For each $B \in Q(A)$ with $\operatorname{rank}(B)=\operatorname{MR}(A), \mathbf{e}_{k} \in \operatorname{rowspace}(B)$ if $k$ is a nonessential index of $A$.

3. Sign patterns that allow diagonalizability. The problem of characterizing sign patterns allowing diagonalizability first came up in the study of sign patterns requiring repeated eigenvalues by Eschenbach and Johnson [6]. Some sufficient conditions for sign patterns to allow diagonalizability were given in [6, 7, 14]. Two characterizations of sign patterns allowing diagonalizability were given in [8] in terms of allowing some more easily checked properties.

If a sign pattern is combinatorially symmetric, then by [14, Theorem 2.6], it allows diagonalizability. In this section, we consider sign patterns whose graphs are trees, but not necessarily combinatorially symmetric. We give some combinatorial structures based on their graphs which are necessary and sufficient for some specific graphs, for example, the star and path to allow diagonalizability. We also give a sufficient condition for a more general class of sign patterns $A$ for which $G(A)$ is a tree to allow diagonalizability.

Let us recall the following definitions from [1, p. 39]. Let $A$ be a square matrix of order $n$. For $1 \leq i, j \leq n$, we say that $i$ has access to $j$ if $i=j$ or there is a path in $D(A)$ from vertex $i$ to vertex $j$, and that $i, j$ communicate if $i$ has access to $j$ and $j$ has access to $i$. This communication relation between the vertices of $D(A)$ is an equivalence relation, which partitions $\{1,2, \ldots, n\}$ into equivalence classes, and each equivalence class will be called a strong component of $D(A)$. We say a strong component $\alpha$ has access to another strong component $\beta$ if $i$ has access to $j$ for all $i \in \alpha$ and for all $j \in \beta$. If $G(A)$ is a tree, then each principal submatrix of $A$ corresponding to a strong component of $D(A)$ is a tree sign pattern matrix.

Throughout this section, $I$ denotes the identity matrix and $|S|$ denotes the cardinality of a finite set $S$.

Lemma 3.1. Let $R$ and $S$ be two square matrices such that $\sigma^{*}(R) \cap \sigma^{*}(S)=\emptyset$. Then for any $X$ of appropriate size, the matrix:

$$
B=\left[\begin{array}{cc}
R & X \\
O & S
\end{array}\right]
$$

is diagonalizable if and only if $\operatorname{rank}(B)=\operatorname{rank}(R)+\operatorname{rank}(S)$ and both $R$ and $S$ are diagonalizable.

Proof. Clearly, $P_{B}(x)=P_{R}(x) P_{S}(x)$. Since $\sigma^{*}(R) \cap \sigma^{*}(S)=\emptyset$, for every $\lambda \in \sigma^{*}(R)$ and $\mu \in \sigma^{*}(S)$,

$$
z(B-\lambda I)=z(R-\lambda I), \quad z(B-\mu I)=z(S-\mu I),
$$

and $\operatorname{nullity}(B-\lambda I)=\operatorname{nullity}(R-\lambda I), \quad \operatorname{nullity}(B-\mu I)=\operatorname{nullity}(S-\mu I)$. 
Electronic Journal of Linear Algebra, ISSN 1081-3810

A publication of the International Linear Algebra Society

Volume 38, pp. 131-159, February 2022.

Further, $\operatorname{rank}(B) \geq \operatorname{rank}(R)+\operatorname{rank}(S)$ and $z(B)=z(R)+z(S)$. Hence, $B$ is diagonalizable if and only if $\operatorname{rank}(B)=\operatorname{rank}(R)+\operatorname{rank}(S)$ and both $R, S$ are diagonalizable.

From the proof of [14, Theorem 2.6], we have the following result.

LEMMA 3.2. If a sign pattern matrix $A$ is combinatorially symmetric, then $c(A)=\operatorname{MR}(A)$.

The following result was conjectured in [9] and proved in [4].

TheOREm 3.3 ([4]). Let $A$ be an $n \times n$ invertible matrix. Then there exists an $n \times n$ invertible diagonal matrix $D$ such that $A D$ has $n$ distinct eigenvalues.

With a minor modification to the proof of Theorem 1.1 in [4], the following result was obtained.

THEOREM 3.4 ([8]). Let $A$ be an $n \times n$ invertible matrix. Then there exists an $n \times n$ invertible diagonal matrix $D$ with positive diagonal entries such that $A D$ has $n$ distinct eigenvalues.

Since the eigenvalues of a matrix depend continuously on its entries, we have the following lemma.

LEMMA 3.5. If $A \in M_{n}$ has an invertible principal submatrix of order $k$ such that all principal submatrices of higher orders are singular, then there are invertible diagonal matrices $D_{1}, D_{2} \in M_{n}$ with positive diagonal entries such that both $D_{1} A$ and $A D_{2}$ have exactly $k$ distinct nonzero eigenvalues.

Proof. Without loss of generality, we may assume that the leading principal minor of $A$ of order $k$ is invertible. Then, we can write $A$ as:

$$
A=\left[\begin{array}{ll}
A_{1} & A_{2} \\
A_{3} & A_{4}
\end{array}\right],
$$

where $A_{1}$ is the leading principal minor of order $k$. So by Lemma 3.4, there exists a diagonal matrix $D$ with positive diagonal entries such that $D A_{1}$ has distinct nonzero eigenvalues. Let $D_{\epsilon}=D \oplus \epsilon I_{n-k}$. Since all principal submatrices of $A$ of order $>k$ are singular, all principal submatrices of $D_{\epsilon} A$ of order $>k$ are singular for all $\epsilon>0$. Again since the eigenvalues of a matrix depend continuously on its entries, we can choose $\epsilon>0$ such that $D_{\epsilon} A$ has exactly $k$ distinct nonzero eigenvalues. Thus, there is an invertible diagonal matrix $D_{1} \in M_{n}$ with positive diagonal entries such that $D_{1} A$ has exactly $k$ distinct nonzero eigenvalues.

Similarly, there exists an invertible diagonal matrix $D_{2} \in M_{n}$ with positive diagonal entries such that $A D_{2}$ has exactly $k$ distinct nonzero eigenvalues.

The following result is from [7, Theorem 2.3].

LEMMA 3.6 ([7]). If a sign pattern matrix $A$ is combinatorially symmetric, then there exists a diagonalizable matrix $B \in Q(A)$ with $\operatorname{rank}(B)=\mathrm{MR}(A)$.

Let $A$ be a sign pattern matrix such that $G(A)$ is a tree. Since $D(A)$ has finitely many strong components, there are strong components $\alpha_{1}, \ldots, \alpha_{l}$ of $D(A)$ such that each $\alpha_{i}$ is accessible only from itself and there are strong components $\beta_{1}, \ldots, \beta_{m}$ of $D(A)$ such that each $\beta_{i}$ has access only to itself. Accordingly, we can represent $D(A)$ as follows. 


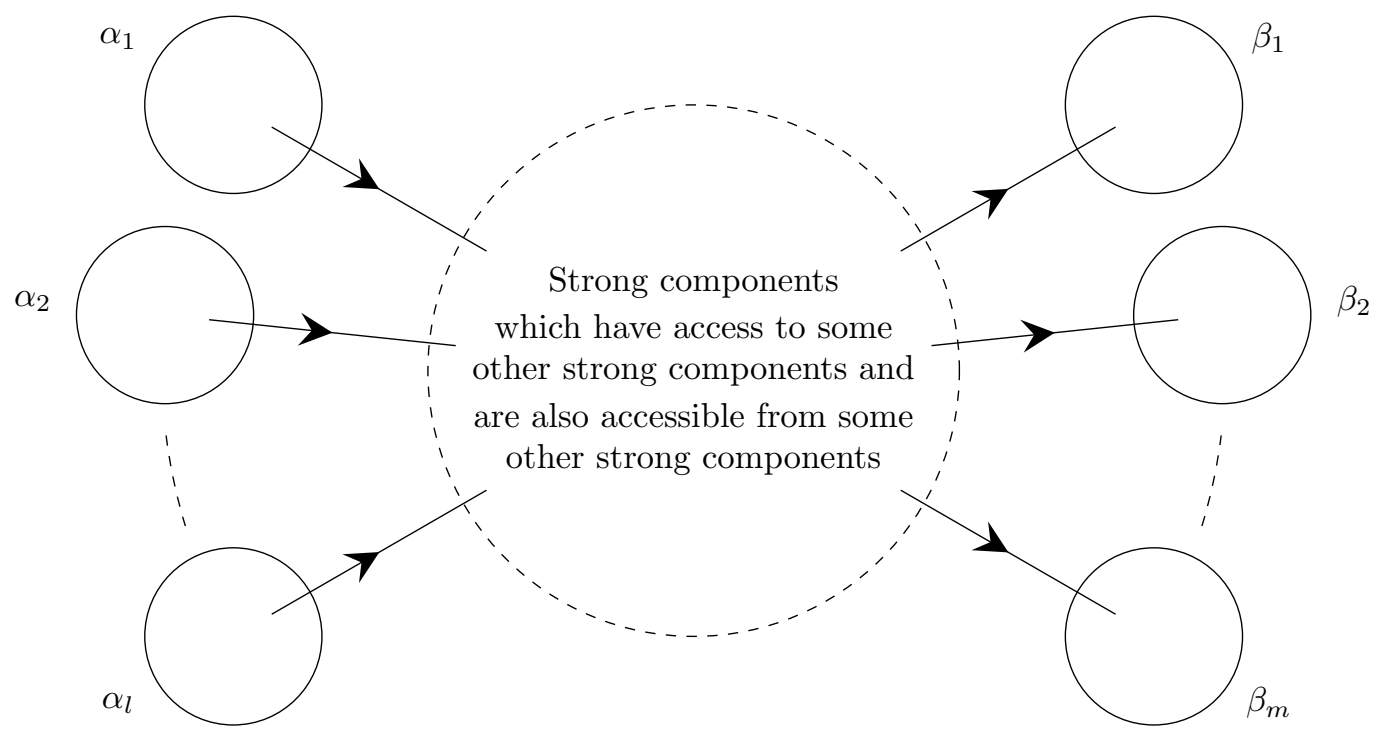

Let $\mathcal{S C}(v)$ be the strong component of $D(A)$ containing $v$. Let $E_{O}$ be a set of essential indices such that $v \in E_{O}$ if and only if there is an $\operatorname{arc}$ in $D(A)$ from $v$ to some vertex $u$ of another strong component of $D(A)$ distinct from $\mathcal{S C}(v)$, and let $E_{I}$ be a set of essential indices such that $v \in E_{I}$ if and only if there is an arc in $D(A)$ to $v$ from some vertex $u$ of another strong component of $D(A)$ distinct from $\mathcal{S C}(v)$. Similarly, let $N_{O}$ be a set of nonessential indices such that $v \in N_{O}$ if and only if there is an arc in $D(A)$ from $v$ to some vertex $u$ of another strong component of $D(A)$ distinct from $\mathcal{S C}(v)$, and let $N_{I}$ be a set of nonessential indices such that $v \in N_{I}$ if and only if there is an arc in $D(A)$ to $v$ from some vertex $u$ of another strong component of $D(A)$ distinct from $\mathcal{S C}(v)$. Let $V(\vec{p})$ denotes the set of all vertices in a directed path $\vec{p}$.

Lemma 3.7. Let $A$ be a sign pattern matrix such that $G(A)$ is a tree. If all possible directed paths between the essential indices of the principal submatrices corresponding to two distinct strong components of $D(A)$ contain nonessential indices from at least one of those strong components, then either there exists $i$ such that $E_{O} \cap \alpha_{i}=\emptyset$ or there exists $j$ such that $E_{I} \cap \beta_{j}=\emptyset$.

Proof. Suppose that $E_{O} \cap \alpha_{i} \neq \emptyset$ for all $i \in\{1,2, \ldots, l\}$, and $E_{I} \cap \beta_{j} \neq \emptyset$ for all $j \in\{1,2, \ldots, m\}$.

Note that for every $r \in\{1,2, \ldots, l\}$, there exists $s \in\{1,2, \ldots, m\}$ such that $\alpha_{r}$ has access to $\beta_{s}$, and for every $s \in\{1,2, \ldots, m\}$, there exists $r \in\{1,2, \ldots, l\}$ such that $\beta_{s}$ has access from $\alpha_{r}$.

Fix $i_{1} \in E_{O} \cap \alpha_{1}$. Since all possible directed paths between the essential indices of the principal submatrices corresponding to two distinct strong components of $D(A)$ contain nonessential indices from at least one of those strong components, there exists a natural number $k$ and vertices $i_{1}, i_{2}, \ldots, i_{4 k+2}$ such that the followings hold, after a possible relabeling of $\alpha$ s and $\beta$ s.

1. For every $t \in\{1,2, \ldots, k+1\}$, there is a directed path $\overrightarrow{p_{t}}$ from $i_{4 t-3} \in E_{O} \cap \alpha_{t}$ to $i_{4 t-2} \in N_{I} \cap \beta_{t}$ such that $\overrightarrow{p_{t}}$ does not contain any vertex from $\mathcal{S C}\left(i_{4 t-3}\right)$ and $\mathcal{S C}\left(i_{4 t-2}\right)$ except $i_{4 t-3}$ and $i_{4 t-2}$, respectively.

2. For every $t \in\{1,2, \ldots, k\}$, there is a directed path $\overrightarrow{q_{t}}$ from $i_{4 t} \in N_{O} \cap \alpha_{t+1}$ to $i_{4 t-1} \in E_{I} \cap \beta_{t}$ such that $\overrightarrow{q_{t}}$ does not contain any vertex from $\mathcal{S C}\left(i_{4 t}\right)$ and $\mathcal{S C}\left(i_{4 t-1}\right)$ except $i_{4 t}$ and $i_{4 t-1}$, respectively.

3. $\alpha_{1}, \alpha_{2}, \ldots, \alpha_{k}$ and $\beta_{1}, \beta_{2}, \ldots, \beta_{k}$ are distinct strong components of $D(A)$.

4. Either $\alpha_{k+1} \in\left\{\alpha_{1}, \alpha_{2}, \ldots, \alpha_{k}\right\}$ or $\beta_{k+1} \in\left\{\beta_{1}, \beta_{2}, \ldots, \beta_{k}\right\}$. 
If either $V\left(\overrightarrow{q_{t}}\right) \cap V\left(\overrightarrow{p_{t}}\right) \neq \emptyset$ or $V\left(\overrightarrow{p_{t+1}}\right) \cap V\left(\overrightarrow{q_{t}}\right) \neq \emptyset$ for some $t \in\{1,2, \ldots, k\}$, then there exists a cycle in $G(A)$ of length at least 3 .

If $V\left(\overrightarrow{q_{t}}\right) \cap V\left(\overrightarrow{p_{t}}\right)=\emptyset$ and $V\left(\overrightarrow{p_{t+1}}\right) \cap V\left(\overrightarrow{q_{t}}\right)=\emptyset$ for all $t \in\{1,2, \ldots, k\}$, then there exists a cycle in $G(A)$ of length at least 4 , since either $\alpha_{k+1} \in\left\{\alpha_{1}, \alpha_{2}, \ldots, \alpha_{k}\right\}$ or $\beta_{k+1} \in\left\{\beta_{1}, \beta_{2}, \ldots, \beta_{k}\right\}$.

In each case, we have a contradiction to the fact that $G(A)$ is a tree.

We know that if a sign pattern matrix $A$ allows nonsingularity or $A$ is combinatorially symmetric, then $A$ allows diagonalizability. The following theorem gives a sufficient condition for a class of sign pattern matrices (not necessarily combinatorially symmetric) that require singularity to allow diagonalizability.

THEOREM 3.8. Let $A$ be a sign pattern matrix such that $G(A)$ is a tree and $A$ requires singularity. If there are no directed paths between the essential indices of the principal submatrices corresponding to two distinct strong components of $D(A)$, or if all possible directed paths between the essential indices of the principal submatrices corresponding to two distinct strong components of $D(A)$ contain nonessential indices from at least one of those strong components, then there exists a diagonalizable matrix $B \in Q(A)$ with $\operatorname{rank}(B)=\operatorname{MR}(A)$.

Proof. We prove this by induction on $r$, the number of distinct strong components of $D(A)$. If $r=1$, then $A$ is combinatorially symmetric and thus by Lemma 3.6, there exists a diagonalizable matrix $B \in Q(A)$ with $\operatorname{rank}(B)=\operatorname{MR}(A)$. Suppose that the theorem is true for any matrix $A$ with the number of distinct strong components of $D(A)$ equal to $r-1$.

Let $A$ be a sign pattern matrix satisfying the given conditions such that the number of distinct strong components of $D(A)$ is $r$.

If there are no directed paths between the essential indices of the principal submatrices corresponding to two distinct strong components of $D(A)$, then either there exists $i$ such that $E_{O} \cap \alpha_{i}=\emptyset$ or there exists $j$ such that $E_{I} \cap \beta_{j}=\emptyset$. If all possible directed paths between the essential indices of the principal submatrices corresponding to two distinct strong components of $D(A)$ contain nonessential indices from at least one of those strong components, then by Lemma 3.7, either there exists $i$ such that $E_{O} \cap \alpha_{i}=\emptyset$ or there exists $j$ such that $E_{I} \cap \beta_{j}=\emptyset$.

Without loss of generality assume that $E_{O} \cap \alpha_{1}=\emptyset$. Since the number of strong components of $D(A)$ is at least $2, N_{O} \cap \alpha_{1} \neq \emptyset$. Let $A_{1}=A\left[\alpha_{1}\right]$ and $\alpha_{1}=\{1,2, \ldots, k\}$. Let $N_{O} \cap \alpha_{1}=\left\{k_{1}, k_{2}, \ldots, k_{t}\right\}$. Then, $k_{1}, k_{2}, \ldots, k_{t}$ are nonessential. Moreover, $A$ can be written as:

$$
A=\left[\begin{array}{cc}
A_{1} & A_{2} \\
O & A_{3}
\end{array}\right]
$$

where the nonzero rows of $A_{2}$ are $k_{1}$-th row, $k_{2}$-th row, and so on up to $k_{t}$-th row. Since $k_{1}, k_{2}, \ldots, k_{t}$ are nonessential for $A_{1}$, by Theorem $2.5, \mathbf{e}_{k_{1}}, \mathbf{e}_{k_{2}}, \ldots, \mathbf{e}_{k_{t}} \in \operatorname{columnspace}\left(B^{\prime}\right)$ for all $B^{\prime} \in Q\left(A_{1}\right)$ with $\operatorname{rank}\left(B^{\prime}\right)=\operatorname{MR}\left(A_{1}\right)$, which implies $\operatorname{MR}(A)=\operatorname{MR}\left(A_{1}\right)+\operatorname{MR}\left(A_{3}\right)$. Using induction hypothesis, we can choose $B_{3} \in Q\left(A_{3}\right)$ such that $B_{3}$ is diagonalizable and $\operatorname{rank}\left(B_{3}\right)=\operatorname{MR}\left(A_{3}\right)$. By Lemma 3.6, we can choose a diagonalizable matrix $B_{1} \in Q\left(A_{1}\right)$ with $\operatorname{rank}\left(B_{1}\right)=\operatorname{MR}\left(A_{1}\right)$ such that $\sigma^{*}\left(B_{1}\right) \cap \sigma^{*}\left(B_{3}\right)=\emptyset$. Since $\mathbf{e}_{k_{1}}, \mathbf{e}_{k_{2}}, \ldots, \mathbf{e}_{k_{t}} \in \operatorname{columnspace}\left(B_{1}\right)$,

$$
B=\left[\begin{array}{cc}
B_{1} & B_{2} \\
O & B_{3}
\end{array}\right] \in Q(A)
$$


$\operatorname{implies} \operatorname{rank}(B)=\operatorname{rank}\left(B_{1}\right)+\operatorname{rank}\left(B_{3}\right)$. Therefore, $\operatorname{rank}(B)=\operatorname{MR}(A)$, and by Lemma 3.1, $B$ is diagonalizable.

In the following example, we show that a sign pattern matrix allows diagonalizability using Theorem 3.8 .

EXAMPLE 3.9. Let

$$
A=\left[\begin{array}{cccccc|ccc|ccc}
0 & 0 & - & 0 & 0 & 0 & 0 & 0 & 0 & 0 & 0 & 0 \\
0 & 0 & + & 0 & 0 & 0 & 0 & 0 & 0 & 0 & 0 & 0 \\
+ & - & + & + & 0 & 0 & 0 & 0 & 0 & 0 & 0 & 0 \\
0 & 0 & - & - & + & 0 & 0 & 0 & 0 & 0 & 0 & 0 \\
0 & 0 & 0 & + & + & + & 0 & 0 & 0 & 0 & 0 & 0 \\
0 & 0 & 0 & 0 & + & 0 & 0 & 0 & 0 & 0 & 0 & 0 \\
\hline 0 & 0 & 0 & 0 & 0 & 0 & 0 & + & 0 & 0 & 0 & 0 \\
0 & 0 & 0 & 0 & 0 & 0 & + & 0 & - & 0 & 0 & 0 \\
0 & 0 & 0 & 0 & 0 & - & 0 & - & 0 & 0 & 0 & 0 \\
\hline 0 & 0 & 0 & 0 & 0 & 0 & 0 & 0 & 0 & 0 & + & 0 \\
0 & 0 & 0 & 0 & 0 & 0 & 0 & 0 & 0 & - & + & - \\
0 & 0 & 0 & 0 & 0 & + & 0 & 0 & 0 & 0 & - & 0
\end{array}\right] .
$$

The digraph $D(A)$ of $A$ is given as follows.

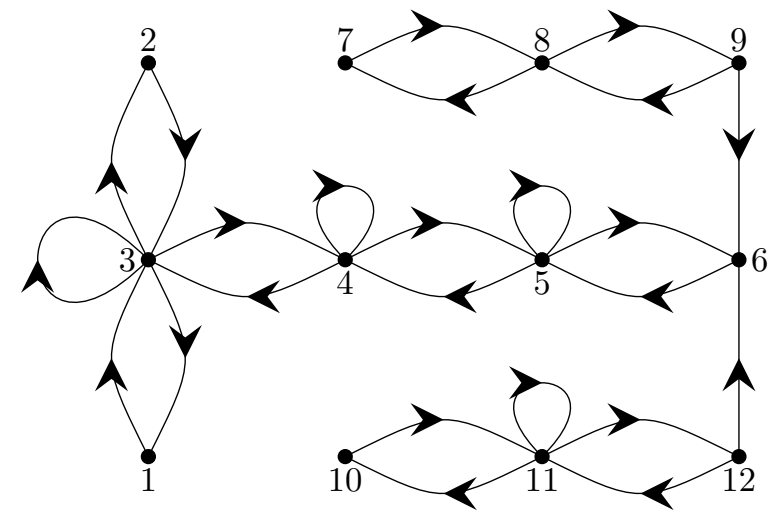

In this example, $G(A)$ is a tree and strong components of $D(A)$ are $\alpha=\{1,2,3,4,5,6\}, \beta=\{7,8,9\}$ and $\gamma=\{10,11,12\}$. Using Example 2.4, we can conclude that the essential indices are 1,2,7,9,10,12. We see that all directed paths from the essential indices in both $\beta$ and $\gamma$ to the essential indices in $\alpha$ contain nonessential indices from $\alpha$. Therefore, there exists $B \in Q(A)$ with $\operatorname{rank}(B)=\operatorname{MR}(A)$.

The following example establishes that the converse of Theorem 3.8 is not true.

EXAMPLE 3.10. Let us consider the sign pattern:

$$
A=\left[\begin{array}{cc|c|c}
0 & + & 0 & + \\
+ & 0 & 0 & 0 \\
\hline+ & 0 & 0 & 0 \\
\hline 0 & 0 & 0 & 0
\end{array}\right]
$$


Electronic Journal of Linear Algebra, ISSN 1081-3810

A publication of the International Linear Algebra Society

Volume 38, pp. 131-159, February 2022.

The digraph $D(A)$ of $A$ is given as follows.

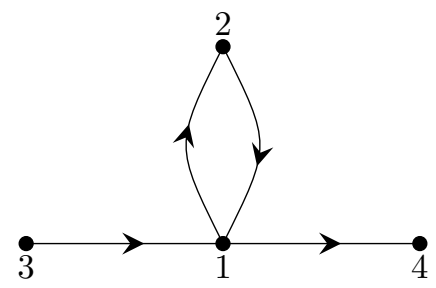

Here, $G(A)$ is a tree and the strong components of $D(A)$ are $\alpha=\{1,2\}, \beta=\{3\}$ and $\gamma=\{4\}$. The essential indices are 3 and 4 . So there is a directed path in $D(A)$ between essential indices of $\beta$ and $\gamma$, namely $3 \rightarrow 1 \rightarrow 4$, containing no other vertex from $\beta$ and $\gamma$. So $A$ does not satisfy the conditions of Theorem 3.8.

Let

$$
B=\left[\begin{array}{ll|l|l}
0 & 1 & 0 & 1 \\
1 & 0 & 0 & 0 \\
\hline 1 & 0 & 0 & 0 \\
\hline 0 & 0 & 0 & 0
\end{array}\right] \in Q(A)
$$

Then, $P_{B}(x)=x^{2}\left(x^{2}-1\right)$. So $\sigma(B)=\{0,1,-1\}$, where $z(0)=2$. Further, nullity $(B)=2$. Therefore, $B$ is diagonalizable.

If we restrict $G(A)$ in Theorem 3.8 to be a path, then the converse is also true.

A matrix of order $n$, whose graph is a path, is permutationally similar to a matrix of the form:

$$
\left[\begin{array}{ccccc}
a_{1} & b_{2} & 0 & \cdots & 0 \\
c_{2} & a_{2} & b_{3} & \ddots & \vdots \\
0 & \ddots & \ddots & \ddots & 0 \\
\vdots & \ddots & c_{n-1} & a_{n-1} & b_{n} \\
0 & \cdots & 0 & c_{n} & a_{n}
\end{array}\right],
$$

where $\left|b_{i}\right|+\left|c_{i}\right| \neq 0$.

A path sign pattern matrix requiring singularity is of the form:

$$
\left[\begin{array}{cccccc}
a_{1} & b_{1} & 0 & \cdots & \ldots & 0 \\
c_{1} & a_{2} & b_{2} & \ddots & & \vdots \\
0 & c_{2} & a_{3} & b_{3} & \ddots & \vdots \\
\vdots & \ddots & \ddots & \ddots & \ddots & 0 \\
\vdots & & \ddots & c_{2 n-1} & a_{2 n} & b_{2 n} \\
0 & \cdots & \cdots & 0 & c_{2 n} & a_{2 n+1}
\end{array}\right],
$$

where $a_{1}=a_{3}=\cdots=a_{2 n+1}=0$ and $b_{i} c_{i} \neq 0$ for $i=1,2, \ldots, 2 n$. In this case, the essential indices are $1,3,5, \ldots, 2 n+1$. 
TheOREm 3.11. Let $A$ be a sign pattern matrix such that $G(A)$ is a path. Then A allows diagonalizability if and only if $A$ allows nonsingularity, or $A$ requires singularity and there are no directed paths between the essential indices of the principal submatrices corresponding to any two distinct strong components of $D(A)$.

Proof. The 'if' part is established by Theorem 3.4 and 3.8. For the 'only if' part, suppose that $A$ requires singularity, and there is a directed path in $D(A)$ between essential indices of two distinct strong components in $D(A)$. Suppose that $A$ has the form (3.11). If $K \in Q(A)$, then we can write

$$
K=\left[\begin{array}{lllll}
X & F & O & O & O \\
G & R & O & O & O \\
O & B & S & O & O \\
O & O & C & T & D \\
O & O & O & E & Y
\end{array}\right]
$$

where $X$ and $Y$ may be vacuous, $R$ and $T$ are matrices of the form (3.12), $S$ is a tridiagonal matrix such that $s_{i+1, i} \neq 0$ for all $i$, exactly one of $F, G$ is a zero matrix, and exactly one of $D$ and $E$ is a zero matrix. Let $R, S$, and $T$ have the orders $n_{1}, n_{2}$, and $n_{3}$, respectively. Since $A$ is of the form (3.11), $b_{1 n_{1}}, c_{1 n_{2}} \neq 0$ and $b_{i j}, c_{i j}=0$ for all other $i, j$. Characteristic polynomial of $K$ is $P_{K}(x)=P_{X}(x) P_{R}(x) P_{S}(x) P_{T}(x) P_{Y}(x)$. Since both $R$ and $T$ are singular, $z(K) \geq z(X)+z(Y)+2$. Further, $\operatorname{rank}(K) \geq \operatorname{rank}(X)+\operatorname{rank}(Y)+n_{1}+n_{2}+n_{3}-1$. Therefore, $\operatorname{nullity}(K) \leq \operatorname{nullity}(X)+\operatorname{nullity}(Y)+1<z(X)+z(Y)+2 \leq z(K)$. So $K$ is not diagonalizable. Thus, $A$ does not allow diagonalizability, a contradiction.

If $G(A)$ is a star, then the converse of Theorem 3.8 is not valid, as it is shown in Example 3.10. However, the following result characterizes all sign pattern matrices whose graph is a star to allow diagonalizability. From [2, p. 294], we note that the Hadamard product of two $m \times n$ matrices $A=\left[a_{i j}\right]$ and $B=\left[b_{i j}\right]$, denoted by $A \circ B$, is defined by $A \circ B=\left[a_{i j} b_{i j}\right]$.

A matrix of order $n$, whose graph is a star, is permutationally similar to a matrix of the form:

$$
\left[\begin{array}{ccccc}
a_{1} & b_{2} & b_{3} & \cdots & b_{n} \\
c_{2} & a_{2} & 0 & \cdots & 0 \\
c_{3} & 0 & a_{3} & \ddots & \vdots \\
\vdots & \vdots & \ddots & \ddots & 0 \\
c_{n} & 0 & \cdots & 0 & a_{n}
\end{array}\right],
$$

where $\left|b_{i}\right|+\left|c_{i}\right| \neq 0$.

TheOREM 3.12. Let $A$ be a sign pattern matrix of the form (3.13) with $n \geq 2$ and $P=\{2,3, \ldots, n\}$. Let $S=\left\{i \in P: a_{i}=0\right\}$ and $T=\left\{i \in P: b_{i} c_{i} \neq 0\right\}$. Then $A$ allows diagonalizability if and only if one of the following conditions hold.

1. $S=\emptyset$.

2. $S \neq \emptyset, T=\emptyset, a_{1} \neq 0$ and either $b_{i}=0$ for all $i \in S$ or $c_{i}=0$ for all $i \in S$.

3. $S, T \neq \emptyset, S \cap T=\emptyset$ and either $b_{i}=0$ for all $i \in S$ or $c_{i}=0$ for all $i \in S$.

4. $S \cap T \neq \emptyset$.

Proof. We first show that if one of the conditions 1, 2, 3, and 4 holds, then $A$ allows diagonalizability. 
Electronic Journal of Linear Algebra, ISSN 1081-3810

A publication of the International Linear Algebra Society

Volume 38, pp. 131-159, February 2022.

151

Sign patterns associated with some graphs that allow or require diagonalizability

1. If $S=\emptyset$, then $A$ allows nonsingularity and thus by Theorem 3.4, $A$ allows diagonalizability.

2. Suppose that $S \neq \emptyset, T=\emptyset, a_{1} \neq 0$ and either $b_{i}=0$ for all $i \in S$ or $c_{i}=0$ for all $i \in S$. We can choose $B \in Q(A)$ such that all nonzero diagonal entries of $B$ are distinct. Since $T=\emptyset$, the eigenvalues of $B$ are its diagonal entries. Since $a_{1} \neq 0, z(B)=|S|=\operatorname{nullity}(B)$. Therefore, $B$ is diagonalizable, and thus $A$ allows diagonalizability.

3. Suppose that $S, T \neq \emptyset, S \cap T=\emptyset$ and either $b_{i}=0$ for all $i \in S$ or $c_{i}=0$ for all $i \in S$. Then $A$ or $A^{T}$ is permutationally similar to

$$
\left[\begin{array}{cc}
A(S) & C \\
O & O
\end{array}\right]
$$

which is of the form (3.13). Since $T \neq \emptyset, A(S)$ allows nonsingularity. So by Theorem 3.4, we can choose $B \in Q(A)$ such that all eigenvalues of $B(S)$ are nonzero and distinct. Further, $z(B)=|S|=$ nullity $(B)$. Therefore $B$ is diagonalizable, and thus $A$ allows diagonalizability.

4. Suppose that $S \cap T \neq \emptyset$. Then, $A$ is permutationally similar to:

$$
\left[\begin{array}{cc}
A[T] & E \\
C & D
\end{array}\right],
$$

which is of the form (3.13) such that $E \circ C^{T}=O$ and $D$ is a diagonal sign pattern matrix. By Lemma 3.5 , there exists $F \in Q(A[T])$ such that all nonzero eigenvalues of $F$ are distinct. Further, there exists $G \in Q(D)$ such that all nonzero diagonal entries of $G$ are distinct and $\sigma^{*}(F) \cap \sigma^{*}(G)=\emptyset$. Let us choose $B \in Q(A)$ such that $B[T]=F$ and $B(T)=G$. Since $E \circ C^{T}=O, P_{B}(x)=P_{B[T]}(x) \cdot P_{B(T)}(x)$ and thus $z(B)=z(B[T])+z(B(T))$. Since $S \cap T \neq \emptyset$, for some $i>1$, the $i$-th column of each one of $B[T], B[T]^{T}$ is a nonzero scalar multiple of $\left[\begin{array}{llll}1 & 0 & \cdots & 0\end{array}\right]^{T}$. Therefore, $\operatorname{rank}(B)=\operatorname{rank}(B[T])+$ $\operatorname{rank}(B(T))$. Further, nullity $(B[T])=|S \cap T|-1=z(B[T])$. So $z(B)=\operatorname{nullity}(B)$. Therefore $B$ is diagonalizable, and thus $A$ allows diagonalizability.

Now we prove the converse part by contradiction. Suppose none of the conditions 1, 2, 3, and 4 are satisfied. Then, we have one of the following.

Case I: $S \neq \emptyset, T=\emptyset$ and $a_{1}=0$.

Case II : $S \cap T=\emptyset$ and there exist $i, j \in S$ such that $b_{i} \neq 0$ and $c_{j} \neq 0$. In this case, $A$ is permutationally similar to

$$
\left[\begin{array}{cc}
A(S) & D \\
C & O
\end{array}\right],
$$

which is of the form (3.13) such that $D \circ C^{T}=O$ and both $C$ and $D$ are nonzero.

In Case I, for every $B \in Q(A), z(B)=|S|+1$, and $\operatorname{rank}(B) \geq n-|S|$. So nullity $(B) \leq|S|<z(B)$. Therefore $B$ is not diagonalizable and hence $A$ does not allow diagonalizability.

In Case II, for every $B \in Q(A), P_{B}(x)=x^{|S|} P_{B(S)}(x)$ and thus $z(B)=|S|+z(B(S))$. Since $\operatorname{rank}(B)=$ $n-|S|+1$, nullity $(B)=|S|-1 \leq z(B(S))+|S|-1<z(B)$ and thus $B$ is not diagonalizable. Hence, $A$ does not allow diagonalizability.

4. Sign patterns that require diagonalizability. If a sign pattern matrix requires all distinct eigenvalues, then it requires diagonalizability. Some necessary and/or sufficient conditions for sign patterns to require distinct eigenvalues can be found in $[5,12,13]$. Lists of sign patterns upto order 4 requiring distinct eigenvalues are given in $[12,13]$.

LEMMA 4.1 ([14]). If $A$ is a sign pattern matrix, then $c(A) \leq \operatorname{MR}(A)$. 
In the following result, we give a necessary condition in terms of the maximum cycle length and the maximum rank for a sign pattern matrix to require diagonalizability.

THEOREM 4.2. If a sign pattern matrix $A$ requires diagonalizability, then $c(A)=\operatorname{MR}(A)$.

Proof. Let $A$ be a sign pattern matrix of order $n$. By Lemma 4.1, $c(A) \leq \mathrm{MR}(A)$. Let $c(A)<\mathrm{MR}(A)$. Let $B \in Q(A)$ be such that $\operatorname{rank}(B)=\mathrm{MR}(A)$. Now $E_{i}(B)=0$ for all $i \geq c(A)+1$, where $E_{i}(B)$ is the sum of all $i \times i$ principal minors of $B$ for $i=1,2, \ldots, n$. Then $x^{n-c(A)}$ is a factor of $P_{B}(x)$. So $\operatorname{nullity}(B)=n-\operatorname{MR}(A)<n-c(A) \leq z(B)$. Therefore, $B$ is not diagonalizable. Hence, $c(A)=\operatorname{MR}(A)$.

The following example shows that the converse of Theorem 4.2 is not true.

EXAMPLE 4.3. Let us consider the sign pattern matrix:

$$
A=\left[\begin{array}{ll}
+ & + \\
- & -
\end{array}\right]
$$

Here $c(A)=\operatorname{MR}(A)=2$. But $A$ does not require diagonalizability, since

$$
B=\left[\begin{array}{cc}
1 & 1 \\
-1 & -1
\end{array}\right] \in Q(A)
$$

and $B$ is not diagonalizable.

In the above example, all terms in the standard determinant expansion of $A$ have different signs. When $c(A)=\operatorname{MR}(A)$, then even the condition "all terms in the standard determinant expansion of $A$ have the same sign" does not necessarily imply that $A$ requires diagonalizability. The following example illustrates this for both reducible and irreducible sign pattern matrices.

EXAMPLE 4.4. Let us consider the sign pattern matrices:

$$
A_{1}=\left[\begin{array}{ll}
+ & + \\
0 & +
\end{array}\right] \text { and } A_{2}=\left[\begin{array}{cc}
+ & + \\
- & +
\end{array}\right] \text {. }
$$

Here, $c\left(A_{1}\right)=\operatorname{MR}\left(A_{1}\right)=2$ and $c\left(A_{2}\right)=\operatorname{MR}\left(A_{2}\right)=2$. But neither $A_{1}$ nor $A_{2}$ require diagonalizability, since

$$
B_{1}=\left[\begin{array}{ll}
1 & 1 \\
0 & 1
\end{array}\right] \in Q\left(A_{1}\right) \text { and } B_{2}=\left[\begin{array}{cc}
4 & 1 \\
-1 & 2
\end{array}\right] \in Q\left(A_{2}\right)
$$

and both $B_{1}$ and $B_{2}$ are non-diagonalizable.

The next few results enable us to reduce the problem of determining star sign pattern matrices, which require diagonalizability for any arbitrary order $n$ to the same problem for the matrices of orders less than or equal to 4 . A signature sign pattern matrix is a diagonal sign pattern matrix whose all diagonal entries are nonzero. A sign pattern matrix $B$ is signature similar to a sign pattern matrix $A$ if there exists a signature sign pattern matrix $S$ such that $B=S A S$. Two sign pattern matrices are said to be equivalent if one is obtained from the other by negation and/or permutation and/or signature similarity. 
Electronic Journal of Linear Algebra, ISSN 1081-3810

A publication of the International Linear Algebra Society

Volume 38, pp. 131-159, February 2022.

Any star sign pattern matrix of order $n$ is permutation and signature similar to a matrix of the form:

$$
S=\left[\begin{array}{ccccc}
a_{1} & + & + & \cdots & + \\
b_{2} & a_{2} & 0 & \cdots & 0 \\
b_{3} & 0 & a_{3} & \ddots & \vdots \\
\vdots & \vdots & \ddots & \ddots & 0 \\
b_{n} & 0 & \cdots & 0 & a_{n}
\end{array}\right]
$$

where $a_{1}, \ldots, a_{n} \in\{+,-, 0\}$ and $b_{2}, \ldots, b_{n} \in\{+,-\}$.

LEMMA 4.5. If a star sign pattern matrix of the form (4.14) requires diagonalizability, then there are no $i, j$ with $i \neq j$ and $i, j \geq 2$ such that $b_{i} \neq b_{j}$ and $a_{i}=a_{j}$.

Proof. Let a star sign pattern matrix $S$ of the form (4.14) be such that $b_{i} \neq b_{j}$ and $a_{i}=a_{j}$ for some $i \neq j$ with $i, j \geq 2$. Suppose that the symbol $a_{i}$ appears multiple times for some $i \geq 2$, and let $\left\{t \geq 2: a_{t}=a_{i}\right\}=\left\{i_{1}, i_{2}, \ldots, i_{k}\right\}$ such that all the symbols $b_{i_{1}}, \ldots, b_{i_{k}}$ are not the same. Therefore, we can choose

$$
B=\left[\begin{array}{ccccc}
\alpha_{1} & 1 & 1 & \ldots & 1 \\
\beta_{2} & \alpha_{2} & 0 & \ldots & 0 \\
\beta_{3} & 0 & \alpha_{3} & \ldots & 0 \\
\vdots & \vdots & \vdots & \ddots & \vdots \\
\beta_{n} & 0 & 0 & \cdots & \alpha_{n}
\end{array}\right] \in Q(S)
$$

such that $\beta_{i_{1}}+\cdots+\beta_{i_{k}}=0$ and $\alpha_{i_{1}}=\cdots=\alpha_{i_{k}}=\alpha_{i}$, say.

The characteristic polynomial of $B$ is

$$
P_{B}(x)=\prod_{p=1}^{n}\left(x-\alpha_{p}\right)-\sum_{p=2}^{n} \beta_{p} \prod_{\substack{q=2 \\ q \neq p}}^{n}\left(x-\alpha_{q}\right) .
$$

Since $\beta_{i_{1}}+\cdots+\beta_{i_{k}}=0,\left(x-\alpha_{i}\right)^{k}$ is a factor of $P_{B}(x)$. So $\alpha_{i}$ is an eigenvalue of $B$ with algebraic multiplicity $\geq k$. Further, $\operatorname{rank}\left(B-\alpha_{i} I\right)=n-k+1$ and thus nullity $\left(B-\alpha_{i} I\right)=k-1$. So $B$ is not diagonalizable. Hence, the result follows.

LEMMA 4.6. Let $B_{1}, B_{2}$ be the matrices given by:

$$
B_{1}=\left[\begin{array}{ccccccc}
\alpha_{1} & 1 & \cdots & \cdots & \cdots & \cdots & 1 \\
\beta_{2} & \alpha_{2} & 0 & \cdots & \cdots & \cdots & 0 \\
\vdots & 0 & \ddots & \ddots & & & \vdots \\
\beta_{k} & \vdots & \ddots & \alpha_{k} & \ddots & & \vdots \\
\beta_{k+1} & \vdots & & \ddots & a & \ddots & \vdots \\
\vdots & \vdots & & & \ddots & \ddots & 0 \\
\beta_{n} & 0 & \cdots & \cdots & \cdots & 0 & a
\end{array}\right] \quad \text { and } B_{2}=\left[\begin{array}{ccccc}
\alpha_{1} & 1 & \cdots & \cdots & 1 \\
\beta_{2} & \alpha_{2} & 0 & \cdots & 0 \\
\vdots & 0 & \ddots & \ddots & \vdots \\
\beta_{k} & \vdots & \ddots & \alpha_{k} & 0 \\
\sum_{n=k+1}^{n} \beta_{i} & 0 & \cdots & 0 & a
\end{array}\right],
$$

where $a \neq \alpha_{i}$ for all $i \in\{2,3, \ldots, k\}$ and $\sum_{i=k+1}^{n} \beta_{i} \neq 0$. Then $B_{1}$ is diagonalizable if and only if $B_{2}$ is diagonalizable. 
Proof. Note that $P_{B_{1}}(x)=(x-a)^{n-k-1} P_{B_{2}}(x)$. If $\lambda \neq a$, then

$$
\operatorname{rank}\left(B_{1}-\lambda I\right)=\operatorname{rank}\left(B_{2}-\lambda I\right)+n-k-1
$$

Further, $\operatorname{rank}\left(B_{1}-a I\right)=\operatorname{rank}\left(B_{2}-a I\right)=k+1$. Moreover, $a \notin \sigma\left(B_{2}\right)$.

Suppose that $B_{1}$ is diagonalizable. If $\lambda \in \sigma\left(B_{2}\right)$, then by $(4.16)$, nullity $\left(B_{1}-\lambda I\right)=\operatorname{nullity}\left(B_{2}-\lambda I\right)$. Further, the algebraic multiplicity of $\lambda$ for $B_{1}$ is same as the algebraic multiplicity of $\lambda$ for $B_{2}$. Hence, $B_{2}$ is diagonalizable.

Suppose that $B_{2}$ is diagonalizable. Let $\lambda \in \sigma\left(B_{1}\right)$. Note that nullity $\left(B_{1}-a I\right)=z\left(B_{1}-a I\right)=n-k-1$. If $\lambda \neq a$, then by (4.16), nullity $\left(B_{1}-\lambda I\right)=\operatorname{nullity}\left(B_{2}-\lambda I\right)$. Further, the algebraic multiplicity of $\lambda$ for $B_{1}$ is same as the algebraic multiplicity of $\lambda$ for $B_{2}$. Hence, $B_{1}$ is diagonalizable.

REMARK 4.7. We use Lemma 4.6 to identify star sign pattern matrices of any order $n$ which does not require diagonalizability by identifying the same for $n=2,3,4$.

We know that if a matrix $A$ belongs to the qualitative class of a symmetric tree sign pattern matrix, then $A$ is similar to a symmetric matrix. If $A$ belongs to the qualitative class of a skew-symmetric tree sign pattern matrix with zero diagonal entries, then $A$ is similar to a skew-symmetric matrix. To identify the star sign pattern matrices that do not require diagonalizability, we need to consider skew-symmetric star sign patterns with some nonzero diagonal entries and the star sign pattern matrices those are neither symmetric nor skew-symmetric.

LEMMA 4.8. No $2 \times 2$ skew-symmetric star sign pattern matrix with some nonzero diagonal entries requires diagonalizability.

Proof. Nonequivalent skew-symmetric star sign pattern matrices of order 2 with some nonzero diagonal entries are

$$
\left[\begin{array}{cc}
0 & + \\
- & -
\end{array}\right],\left[\begin{array}{ll}
- & + \\
- & -
\end{array}\right],\left[\begin{array}{ll}
+ & + \\
- & -
\end{array}\right]
$$

None of the above sign pattern matrices requires diagonalizability, and examples of non-diagonalizable matrices in their qualitative classes are respectively

$$
\left[\begin{array}{cc}
0 & 1 \\
-1 & -2
\end{array}\right],\left[\begin{array}{cc}
-1 & 1 \\
-1 & -3
\end{array}\right],\left[\begin{array}{cc}
1 & 1 \\
-1 & -1
\end{array}\right]
$$

These are non-diagonalizable because their characteristic polynomials are respectively

$$
(x+1)^{2}, \quad(x+2)^{2}, \quad x^{2},
$$

whereas all the eigenvalues have geometric multiplicity 1.

LEMMA 4.9. No $3 \times 3$ skew-symmetric star sign pattern matrix with some nonzero diagonal entries requires diagonalizability.

Proof. Non-equivalent skew-symmetric star sign pattern matrices of order 3 with some nonzero diagonal entries are

$$
\left[\begin{array}{ccc}
0 & + & + \\
- & - & 0 \\
- & 0 & -
\end{array}\right],\left[\begin{array}{ccc}
- & + & + \\
- & - & 0 \\
- & 0 & -
\end{array}\right],\left[\begin{array}{ccc}
+ & + & + \\
- & - & 0 \\
- & 0 & -
\end{array}\right],\left[\begin{array}{ccc}
- & + & + \\
- & 0 & 0 \\
- & 0 & 0
\end{array}\right],
$$


Electronic Journal of Linear Algebra, ISSN 1081-3810

A publication of the International Linear Algebra Society

Volume 38, pp. 131-159, February 2022.

$$
\left[\begin{array}{ccc}
0 & + & + \\
- & - & 0 \\
- & 0 & 0
\end{array}\right],\left[\begin{array}{ccc}
- & + & + \\
- & - & 0 \\
- & 0 & 0
\end{array}\right],\left[\begin{array}{ccc}
+ & + & + \\
- & - & 0 \\
- & 0 & 0
\end{array}\right],\left[\begin{array}{ccc}
- & + & + \\
- & - & 0 \\
- & 0 & +
\end{array}\right],\left[\begin{array}{ccc}
0 & + & + \\
- & - & 0 \\
- & 0 & +
\end{array}\right] .
$$

None of the first four sign pattern matrices from the above list requires diagonalizability because we can find non-diagonalizable matrices in their qualitative classes using Lemmas 4.6 and 4.8. None of the next five sign pattern matrices from the above list requires diagonalizability, and examples of non-diagonalizable matrices in their qualitative classes are respectively

$$
\left[\begin{array}{ccc}
0 & 6 & 3 \\
-4 & -9 & 0 \\
-1 & 0 & 0
\end{array}\right],\left[\begin{array}{ccc}
-2 & 1 & 1 \\
-2 & -4 & 0 \\
-2 & 0 & 0
\end{array}\right],\left[\begin{array}{ccc}
2 & 9 & 1 \\
-3 & -8 & 0 \\
-1 & 0 & 0
\end{array}\right],\left[\begin{array}{ccc}
-1 & 1 & 1 \\
-2 & -3 & 0 \\
-2 & 0 & 1
\end{array}\right],\left[\begin{array}{ccc}
0 & 1 & 1 \\
-2 & -2 & 0 \\
-2 & 0 & 2
\end{array}\right]
$$

These are non-diagonalizable because their characteristic polynomials are respectively

$$
(x+3)^{3}, \quad(x+2)^{3}, \quad(x+2)^{3}, \quad(x+1)^{3}, \quad x^{3},
$$

whereas all the eigenvalues have geometric multiplicity 1 .

LEMma 4.10. No $3 \times 3$ star sign pattern matrix, which is neither symmetric nor skew-symmetric, requires diagonalizability.

Proof. Each matrix in the qualitative class of such a sign pattern matrix is equivalent to a matrix of the form:

$$
\left[\begin{array}{ccc}
\alpha_{1} & 1 & 1 \\
-\beta_{2} & \alpha_{2} & 0 \\
\beta_{3} & 0 & \alpha_{3}
\end{array}\right]
$$

where $\beta_{2}, \beta_{3}>0$ and $\alpha_{1}, \alpha_{2}, \alpha_{3} \in \mathbb{R}$. If the qualitative class of a sign pattern matrix contains a matrix of this form such that $\operatorname{sgn}\left(\alpha_{2}\right)=\operatorname{sgn}\left(\alpha_{3}\right)$, then by Lemma 4.5, that sign pattern matrix does not require diagonalizability. Now the remaining nonequivalent sign pattern matrices of the form (4.17) are

$$
\begin{gathered}
{\left[\begin{array}{ccc}
0 & + & + \\
- & - & 0 \\
+ & 0 & 0
\end{array}\right],\left[\begin{array}{ccc}
+ & + & + \\
- & - & 0 \\
+ & 0 & 0
\end{array}\right],\left[\begin{array}{ccc}
- & + & + \\
- & - & 0 \\
+ & 0 & 0
\end{array}\right],\left[\begin{array}{ccc}
0 & + & + \\
- & 0 & 0 \\
+ & 0 & +
\end{array}\right],\left[\begin{array}{ccc}
- & + & + \\
- & 0 & 0 \\
+ & 0 & +
\end{array}\right],\left[\begin{array}{ccc}
+ & + & + \\
- & 0 & 0 \\
+ & 0 & +
\end{array}\right],} \\
{\left[\begin{array}{ccc}
+ & + & + \\
- & - & 0 \\
+ & 0 & +
\end{array}\right],\left[\begin{array}{ccc}
- & + & + \\
- & - & 0 \\
+ & 0 & +
\end{array}\right],\left[\begin{array}{lll}
0 & + & + \\
- & - & 0 \\
+ & 0 & +
\end{array}\right] .}
\end{gathered}
$$

None of the above sign pattern matrices requires diagonalizability, and examples of non-diagonalizable matrices in their qualitative classes are respectively

$$
\begin{gathered}
{\left[\begin{array}{ccc}
0 & 3 & 5 \\
-8 & -1 & 0 \\
9 & 0 & 0
\end{array}\right],\left[\begin{array}{ccc}
1 & 1 & 1 \\
-5 & -4 & 0 \\
1 & 0 & 0
\end{array}\right],\left[\begin{array}{ccc}
-1 & 1 & 4 \\
-3 & -1 & 0 \\
2 & 0 & 0
\end{array}\right],\left[\begin{array}{ccc}
0 & 7 & 8 \\
-9 & 0 & 0 \\
12 & 0 & 1
\end{array}\right],\left[\begin{array}{ccc}
-2 & 9 & 8 \\
-5 & 0 & 0 \\
8 & 0 & 1
\end{array}\right],\left[\begin{array}{ccc}
5 & 1 & 1 \\
-5 & 0 & 0 \\
1 & 0 & 4
\end{array}\right],} \\
{\left[\begin{array}{ccc}
2 & 1 & 1 \\
-5 & -3 & 0 \\
1 & 0 & 1
\end{array}\right],\left[\begin{array}{ccc}
-1 & 4 & 4 \\
-3 & -1 & 0 \\
8 & 0 & 1
\end{array}\right],\left[\begin{array}{ccc}
0 & 5 & 6 \\
-4 & -12 & 0 \\
6 & 0 & 4
\end{array}\right] .}
\end{gathered}
$$


These are non-diagonalizable because their characteristic polynomials are respectively

$$
\begin{gathered}
(x+3)^{2}(x-5), \quad(x+2)^{2}(x-1), \quad(x+2)^{2}(x-2), \quad(x+3)^{2}(x-7), \quad(x+3)^{2}(x-5), \\
(x-2)^{2}(x-5), \quad(x-2)(x+1)^{2}, \quad(x+3)^{2}(x-5), \quad(x+8)^{2}(x-8),
\end{gathered}
$$

whereas all the eigenvalues have geometric multiplicity 1 .

Lemma 4.11. No $4 \times 4$ skew-symmetric star sign pattern matrix with some nonzero diagonal entries requires diagonalizability.

Proof. Each matrix in the qualitative class of such a sign pattern matrix is equivalent to a matrix of the form:

$$
\left[\begin{array}{cccc}
\alpha_{1} & 1 & 1 & 1 \\
-\beta_{2} & \alpha_{2} & 0 & 0 \\
-\beta_{3} & 0 & \alpha_{3} & 0 \\
-\beta_{4} & 0 & 0 & \alpha_{4}
\end{array}\right]
$$

where $\beta_{2}, \beta_{3}, \beta_{4}>0$ and $\alpha_{1}, \alpha_{2}, \alpha_{3}, \alpha_{4} \in \mathbb{R}$. If the qualitative class of a sign pattern matrix contains a matrix of this form such that any two of $\operatorname{sgn}\left(\alpha_{2}\right), \operatorname{sgn}\left(\alpha_{3}\right), \operatorname{sgn}\left(\alpha_{4}\right)$ are equal, then by Lemma 4.6, 4.8, and 4.9, that sign pattern matrix does not require diagonalizability. Now the remaining nonequivalent sign pattern matrices of the form (4.18) are

$$
\left[\begin{array}{cccc}
+ & + & + & + \\
- & - & 0 & 0 \\
- & 0 & 0 & 0 \\
- & 0 & 0 & +
\end{array}\right],\left[\begin{array}{cccc}
0 & + & + & + \\
- & - & 0 & 0 \\
- & 0 & 0 & 0 \\
- & 0 & 0 & +
\end{array}\right]
$$

None of the above sign pattern matrices requires diagonalizability, and examples of non-diagonalizable matrices in their qualitative classes are respectively

$$
\left[\begin{array}{cccc}
5 & 5 & 5 & 5 \\
-4 & -5 \sqrt{5} & 0 & 0 \\
-2 & 0 & 0 & 0 \\
-4 & 0 & 0 & 5 \sqrt{5}
\end{array}\right],\left[\begin{array}{cccc}
0 & 10 & 4 & 21 \\
-25 & -32 & 0 & 0 \\
-3 & 0 & 0 & 0 \\
-18 & 0 & 0 & 32
\end{array}\right] .
$$

These are non-diagonalizable because their characteristic polynomials are respectively

$$
(x-5)^{3}(x+10), \quad(x-8)^{3}(x+24),
$$

whereas all the eigenvalues have geometric multiplicity 1.

LEMMA 4.12. No $4 \times 4$ star sign pattern matrix, which is neither symmetric nor skew-symmetric, requires diagonalizability.

Proof. Each matrix in the qualitative class of such a sign pattern matrix is equivalent to a matrix of the form:

$$
\left[\begin{array}{cccc}
\alpha_{1} & 1 & 1 & 1 \\
-\beta_{2} & \alpha_{2} & 0 & 0 \\
\beta_{3} & 0 & \alpha_{3} & 0 \\
\beta_{4} & 0 & 0 & \alpha_{4}
\end{array}\right] \quad \text { or } \quad\left[\begin{array}{cccc}
\alpha_{1} & 1 & 1 & 1 \\
-\beta_{2} & \alpha_{2} & 0 & 0 \\
-\beta_{3} & 0 & \alpha_{3} & 0 \\
\beta_{4} & 0 & 0 & \alpha_{4}
\end{array}\right] \text {, }
$$

where $\beta_{2}, \beta_{3}, \beta_{4}>0$ and $\alpha_{1}, \alpha_{2}, \alpha_{3}, \alpha_{4} \in \mathbb{R}$. If the qualitative class of a sign pattern matrix contains a matrix of the first form, then we have the following. 
Electronic Journal of Linear Algebra, ISSN 1081-3810

A publication of the International Linear Algebra Society

Volume 38, pp. 131-159, February 2022.

1. If $\operatorname{sgn}\left(\alpha_{2}\right)$ is equal to one of $\operatorname{sgn}\left(\alpha_{3}\right), \operatorname{sgn}\left(\alpha_{4}\right)$, then by Lemma 4.5, that sign pattern matrix does not require diagonalizability.

2. If $\operatorname{sgn}\left(\alpha_{3}\right)=\operatorname{sgn}\left(\alpha_{4}\right)$, then by Lemmas 4.6 and 4.10 , that sign pattern matrix does not require diagonalizability.

Similarly, if the qualitative class of a sign pattern matrix contains a matrix of the second form, then we have the following.

1. If $\operatorname{sgn}\left(\alpha_{4}\right)$ is equal to one of $\operatorname{sgn}\left(\alpha_{2}\right), \operatorname{sgn}\left(\alpha_{3}\right)$, then by Lemma 4.5, that sign pattern matrix does not require diagonalizability.

2. If $\operatorname{sgn}\left(\alpha_{2}\right)=\operatorname{sgn}\left(\alpha_{3}\right)$, then by Lemmas 4.6 and 4.10 , that sign pattern matrix does not require diagonalizability.

So the remaining nonequivalent sign pattern matrices of the above forms are

$$
\begin{aligned}
& {\left[\begin{array}{cccc}
+ & + & + & + \\
- & - & 0 & 0 \\
+ & 0 & 0 & 0 \\
+ & 0 & 0 & +
\end{array}\right],\left[\begin{array}{cccc}
0 & + & + & + \\
- & - & 0 & 0 \\
+ & 0 & 0 & 0 \\
+ & 0 & 0 & +
\end{array}\right],\left[\begin{array}{cccc}
- & + & + & + \\
- & - & 0 & 0 \\
+ & 0 & 0 & 0 \\
+ & 0 & 0 & +
\end{array}\right],\left[\begin{array}{cccc}
+ & + & + & + \\
- & 0 & 0 & 0 \\
+ & 0 & - & 0 \\
+ & 0 & 0 & +
\end{array}\right],\left[\begin{array}{cccc}
0 & + & + & + \\
- & 0 & 0 & 0 \\
+ & 0 & - & 0 \\
+ & 0 & 0 & +
\end{array}\right],} \\
& {\left[\begin{array}{cccc}
+ & + & + & + \\
- & - & 0 & 0 \\
- & 0 & 0 & 0 \\
+ & 0 & 0 & +
\end{array}\right],\left[\begin{array}{cccc}
0 & + & + & + \\
- & - & 0 & 0 \\
- & 0 & 0 & 0 \\
+ & 0 & 0 & +
\end{array}\right],\left[\begin{array}{cccc}
- & + & + & + \\
- & - & 0 & 0 \\
- & 0 & 0 & 0 \\
+ & 0 & 0 & +
\end{array}\right],\left[\begin{array}{llll}
+ & + & + & + \\
- & - & 0 & 0 \\
- & 0 & + & 0 \\
+ & 0 & 0 & 0
\end{array}\right],\left[\begin{array}{cccc}
0 & + & + & + \\
- & - & 0 & 0 \\
- & 0 & + & 0 \\
+ & 0 & 0 & 0
\end{array}\right] .}
\end{aligned}
$$

None of the above sign pattern matrices requires diagonalizability, and examples of non-diagonalizable matrices in their qualitative classes are respectively

$$
\begin{aligned}
& {\left[\begin{array}{cccc}
105 & 84 & 21 & 21 \\
-81 & -84 & 0 & 0 \\
14 & 0 & 0 & 0 \\
16 & 0 & 0 & 63
\end{array}\right],\left[\begin{array}{cccc}
0 & 10 & 24 & 21 \\
-9 & -18 & 0 & 0 \\
32 & 0 & 0 & 0 \\
14 & 0 & 0 & 18
\end{array}\right],\left[\begin{array}{cccc}
-36 & 21 & 72 & 21 \\
-30 & -54 & 0 & 0 \\
64 & 0 & 0 & 0 \\
42 & 0 & 0 & 54
\end{array}\right]} \\
& {\left[\begin{array}{cccc}
12 & 8 & 9 & 2 \\
-16 & 0 & 0 & 0 \\
10 & 0 & -2 & 0 \\
9 & 0 & 0 & 2
\end{array}\right],\left[\begin{array}{cccc}
0 & 16 & 18 & 2 \\
-12 & 0 & 0 & 0 \\
15 & 0 & -2 & 0 \\
7 & 0 & 0 & 2
\end{array}\right],\left[\begin{array}{cccc}
5 & 5 & 5 & 5 \\
-42 & -25 & 0 & 0 \\
-8 & 0 & 0 & 0 \\
15 & 0 & 0 & 5
\end{array}\right],} \\
& {\left[\begin{array}{cccc}
0 & 10 & 10 & 9 \\
-9 & -20 & 0 & 0 \\
-5 & 0 & 0 & 0 \\
10 & 0 & 0 & 5
\end{array}\right],\left[\begin{array}{cccc}
-6 & 6 & 12 & 9 \\
-5 & -18 & 0 & 0 \\
-8 & 0 & 0 & 0 \\
18 & 0 & 0 & 6
\end{array}\right],\left[\begin{array}{cccc}
4 & 1 & 1 & 1 \\
-2 & -\sqrt{3} & 0 & 0 \\
-2 & 0 & \sqrt{3} & 0 \\
3 & 0 & 0 & 0
\end{array}\right],\left[\begin{array}{cccc}
0 & 2 & 2 & 2 \\
-9 & -8 & 0 & 0 \\
-9 & 0 & 8 & 0 \\
2 & 0 & 0 & 0
\end{array}\right] .}
\end{aligned}
$$

These are non-diagonalizable because their characteristic polynomials are respectively

$$
\begin{gathered}
(x+21)^{2}(x-42)(x-84), \quad(x+24)^{2}(x-12)(x-36), \quad(x+72)^{2}(x-72)(x-36), \\
(x-8)(x-4)^{2}(x+4), \quad(x-4)^{3}(x+12), \quad(x+10)^{2}(x+5)(x-10), \\
(x+10)^{2}(x+5)(x-10), \quad(x+12)^{2}(x+6)(x-12), \quad(x-3)^{2}(x+1)^{2}, \quad(x-4)^{2}(x+4)^{2},
\end{gathered}
$$

and all the eigenvalues have geometric multiplicity 1. 
The following result gives a complete description of star sign pattern matrices that require diagonalizability.

THEOREM 4.13. A star sign pattern matrix $S$ requires diagonalizability if and only if $S$ is a symmetric sign pattern matrix or a skew-symmetric sign pattern matrix with all diagonal entries zero.

Proof. If $S$ is either a symmetric star sign pattern matrix or a skew-symmetric star sign pattern matrix with all diagonal entries zero, then every matrix in $Q(S)$ is similar to either a symmetric matrix or a skew-symmetric matrix. So $S$ requires diagonalizability.

For the converse part, suppose that $S$ is neither a symmetric sign pattern matrix nor a skew-symmetric sign pattern matrix with all diagonal entries zero. Suppose that $S$ is of the form (4.14). If there are $i$ and $j$ with $i \neq j$ and $i, j \geq 2$ such that $b_{i} \neq b_{j}$ and $a_{i}=a_{j}$, then by Lemma 4.5, $S$ does not require diagonalizability. Otherwise, that is if $S$ satisfies the condition that $a_{i}=a_{j}$ implies $b_{i}=b_{j}$ for all $i, j \geq 2$, then we can find a non-diagonalizable matrix in $Q(S)$ using Lemma 4.6 and one of Lemma 4.8, 4.9, 4.10, 4.11, and 4.12. So $S$ does not require diagonalizability.

The following example shows that Theorem 4.13 cannot be extended to the path sign pattern matrices.

EXAMPLE 4.14. Let us consider the path sign pattern matrix:

$$
\left[\begin{array}{cccc}
0 & + & 0 & 0 \\
- & 0 & + & 0 \\
0 & + & 0 & + \\
0 & 0 & + & 0
\end{array}\right],
$$

which is neither symmetric nor skew-symmetric. Any matrix in its qualitative class is similar to a matrix of the form:

$$
B=\left[\begin{array}{cccc}
0 & 1 & 0 & 0 \\
-a & 0 & 1 & 0 \\
0 & b & 0 & 1 \\
0 & 0 & c & 0
\end{array}\right]
$$

where $a, b, c>0$. The characteristic polynomial of $B$ is

$$
\begin{aligned}
P_{B}(x) & =x^{4}+(a-b-c) x^{2}-a c \\
& =\left(x^{2}-\frac{b+c-a+\sqrt{(b+c-a)^{2}+4 a c}}{2}\right)\left(x^{2}-\frac{b+c-a-\sqrt{(b+c-a)^{2}+4 a c}}{2}\right) .
\end{aligned}
$$

So $B$ has one positive, one negative, and two purely imaginary eigenvalues and thus $B$ is diagonalizable. Hence, the above sign pattern matrix requires diagonalizability.

Acknowledgment. I am grateful to Indian Institute of Technology Guwahati, India, for providing me with a graduate fellowship to carry out research. I thank Dr. Sriparna bandyopadhyay for careful reading and suggestions for a better presentation of the paper. I am also grateful to the reviewer(s) for their comments and suggestions which help to improve the paper and thankful to the Editor-in-Chief for giving me a chance to revise. 


\section{REFERENCES}

[1] A. Berman and R. J. Plemmons. Nonnegative Matrices in the Mathematical Sciences. SIAM, Philadelphia, 1994.

[2] R.A. Brualdi and H.J. Ryser. Combinatorial Matrix Theory. Cambridge University Press, Cambridge, 1991.

[3] R.A. Brualdi and B.L. Shader. Matrices of Sign-Solvable Linear Systems. Vol. 116. Cambridge Tracts in Mathematics, Cambridge University Press, Cambridge, 1995.

[4] M. Choi, Z. Huang, C. Li, and N. Sze. Every invertible matrix is diagonally equivalent to a matrix with distinct eigenvalues. Linear Algebra Appl., 436(9):3773-3776, 2012.

[5] C.A. Eschenbach, F.J. Hall, and Z. Li. Eigenvalue frequency and consistent sign pattern matrices. Czechoslovak Math. J., 44:461-479, 1994.

[6] C.A. Eschenbach and C.R. Johnson. Sign patterns that require repeated eigenvalues. Linear Algebra Appl., 190:161-179, 1993.

[7] X. Feng, W. Gao, F.J. Hall, G. Jing, Z. Li, C. Zagrodny, and J. Zhou. Rank conditions for sign patterns that allow diagonalizability. Discrete Math., 343(5):111798, 11 pp., 2020.

[8] X. Feng, T. Huang, Z. Li, J. Luo, and Y. Gao. Sign patterns that allow diagonalizability revisited. Linear Multilinear Algebra 61(9):1223-1233, 2013.

[9] X. Feng, Z. Li, and T. Huang. Is every nonsingular matrix is diagonally equivalent to a matrix with all distinct eigenvalues? Linear Algebra Appl. 436:120-125, 2012.

[10] F.J. Hall and Z. Li. Sign Pattern Matrices. In: L. Hogben (editor), Handbook of Linear Algebra. 2nd edition. CRC Press, Boca Raton, FL, 2014.

[11] C. Jeffries and C.R. Johnson. Some sign patterns that preclude matrix stability. SIAM J. Matrix Anal. Appl., 9(1):19-25, 1988.

[12] P.J. Kim. On the 4 by 4 Irreducible Sign Pattern Matrices that Require Four Distinct Eigenvalues. Thesis, Georgia State University, 2011. Available at: https://scholarworks.gsu.edu/math_theses/101/

[13] Z. Li and L. Harris. Sign patterns that require all distinct eigenvalues. JP J. Algebra Number Theory Appl., 2(2):161-179, 2002.

[14] Y. Shao and Y. Gao. Sign patterns that allow diagonalizability. Linear Algebra Appl., 359:113-119, 2003. 